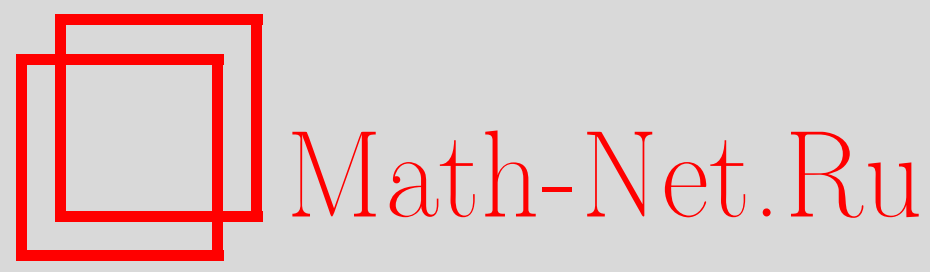

М. Саралеги-Арангурен, Р. Волак, Базовые когомологии пересечения конических расслоений, Матем. заметки, 2005, том 77, выпуск 2, 235-257

DOI: https://doi.org/10.4213/mzm2487

Использование Общероссийского математического портала Math-Net.Ru подразумевает, что вы прочитали и согласны с пользовательским соглашением http://www.mathnet.ru/rus/agreement

Параметры загрузки:

IP : 54.157 .27 .8

26 апреля 2023 г., 18:26:50

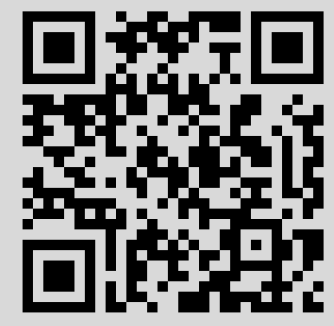




\title{
БАЗОВЫЕ КОГОМОЛОГИИ ПЕРЕСЕЧЕНИЯ КОНИЧЕСКИХ РАССЛОЕНИЙ
}

\author{
М. Саралеги-Арангурен, Р. Волак
}

\begin{abstract}
Вводится понятие сингулярного расслоения и сингулярного расслоения Зейферта. Эти понятия естественно обобщают понятие локальо тривиального расслоения на случай категории стратифицированных псевдомногообразий. Для сингулярных слоений, определяемых такими расслоениями, мы доказываем теорему типа де Рама для базовых когомологий пересечений, введенных недавно авторами. Одним из важных примеров такой структуры является естественная проекция на пространство слоев сингулярного риманова слоения, определяемого действием компактной группы Ли на гладком компактном многообразии.

Библиография: 23 названия.
\end{abstract}

\section{1. Введение}

Отсутствие двойственности Пуанкаре для гомологий и когомологий некоторых сингулярных пространств привело Горески и Макферсона к введению новой теории гомологий, названной гомологиями пересечений, которая учитывает сингулярности рассматриваемого пространства (см. [1]).

Эти гомологии определяются на стратифицированных псевдомногообразиях. Начальная идея обобщалась в различных направлениях. Развивалась теория симплищиальных и сингулярных гомологий, а также были предложены более слабые понятия искажения (дифференциальных форм) (см. [2], [3]).

Было установлено несколько версий двойственности Пуанкаре с учетом степени искажения. Наконец, Горески и Макферсон определили когомологии де Рама пересечений для стратифицированных пространств в смысле Тома-Мезера. Однако они не опубликовали своего определения, и позже это определение было включено в работу Брылински (см. [4]) об эквивариантных когомологиях. Все это привело к поиску теоремы типа де Рама для когомологий пересечений.

Работа первого автора выполнена при частичной поддержке программ "Programa de perfeccionamiento y movilidad de personal investigador" фонда "Eusko Jaularitza-Gobierno del País Vasco" и "Projet d'Action Integrée Picasso", грант № 02679SL. Работа второго автора выполнена при частичной поддержке фонда КBN, грант № 5 P03A 04020. 
Первьй автор в его диссертации и нескольких последовательных публикациях (см. [5]) рассмотрел когогомологии де Рама пересечений для стратифищированного пространства, существенно используя разрешение сингулярностей по Верону (см. [6]). Главной идеей явилось введение комплекса (пучка) форм пересечения, допускаюших поднятие (см. [5]).

В работе [7] авторы ввели базовые когомологии пересечения (в смысле де Рама), или сокращенно БКП, для сингулярных римановых слоений, используя тот факт, что такие слоения определяют стратификацию многообразия (см. [8], [9]) по отношению к слоям слоения. На самом деле, когомологии БКП можно определить для более широкого класса слоений, которые мы назьваем коническими слоениями.

В настоящей работе мы изучаем когомологии БКП для специального класса конических слоений, задаваемых так называемыми сингулярными расслоениями. В этом случае пространство слоев является стратифишированным псевдомногообразием, а когомологии БКП этого слоения совпадают с когомологиями пересечений пространства слоев. В случае, когда сингулярное расслоение в действительности является регулярным, мы получаем хорошо известную теорему де Рама для многообразий.

\section{2. Конические слоения}

2.1. Сингулярные слоения. Слоение $\mathscr{F}$ многообразия $M$ - это разбиение многообразия $M$ на гладкие связные подмногообразия, называемые слоями, которые необязательно вложены. Мы вводим только одно ограничение на это разбиение, связанное с теоремой Фробениуса (о различных версиях теоремы Фробениуса см. [7], [10]-[15]). Полезно различать два случая: регулярньй и сингулярный.

Разбиение $\mathscr{F}$ называется регулярным слоением размерности $p$, если подмногообразия разбиения имеют одну и ту же размерность $p$ и для любой точки $m \in M$ сушествует такая карта $(U, \varphi), \varphi=\left(x_{1}, \ldots, x_{p}, y_{1}, \ldots, y_{q}\right)$, что слои

$$
\varphi_{c}=\left\{p \in U \mid\left(y_{1}(p), \ldots, y_{q}(p)\right)=c\right\}, \quad c \in \mathbb{R}^{q},
$$

являются связными компонентами следов слоев на карте $U$. Поэтому локально слоение $\mathscr{F}$ представляется произведением

$$
\left(\mathbb{R}^{m}, \mathscr{H}\right) \equiv\left(\mathbb{R}^{p} \times \mathbb{R}^{q}, \mathscr{J} \times \mathscr{I}\right),
$$

причем $\mathscr{J}$ является слоением пространства $\mathbb{R}^{p}$ ровно с одним слоем, а $\mathscr{I}$ является слоением пространства $\mathbb{R}^{q}$, состоящим из отдельньх точек. Слоение $\mathscr{H}$ называется $n р о с-$ тым мл лоением.

Теорема Фробениуса приводит нас к следующему эквивалентному определению регулярного слоения.

Пусть D обозначает векторное подрасслоение в ТM постоянного ранга. Тогда $D$ называется (регулярным) слоением, если оно является инволютивным подрасслоением, т.е. если для любых векторных полей $X$ и $Y$, являющихся сечениями подрасслоения $D$, их скобка Ли $[X, Y]$ тоже является сечением подрасслоения $D$.

Эквивалентность этих двух определений заключается в том, что инволютивному подрасслоению сопоставляется разбиение на максимальные интегральные подмногообразия, которых касаются векторы из подрасслоения. В сингулярном случае мы получаем более деликатное условие. 
Рассмотрим распределение $D \subset T M$ такое, что для любой точки $x \in M$ существует открытая окрестность $U$ точки $x$ и гладкие векторные поля $X_{1}, \ldots, X_{k}$ на $U$, порождаюшие $D_{y}$ в каждой точке $y \in U$. Распределение $D$ называется сингулярным слоением, если

1) подрасслоение $D$ инволютивно;

2 ) если $X$ есть локальное сечение распределения $D$, а $\varphi_{t}$ - его локальньй поток, то размерность $D$ постоянна вдоль каждой траектории потока $\varphi_{t}$.

Сингулярная теорема Фробениуса утверждает, что эти условия эквивалентны следующему условию.

Пусть слой $L_{m}$, проходящий через точку $m \in M$, имеет размерность $p$. Тогда существует такая карта $(U, \varphi), \varphi=\left(x_{1}, \ldots, x_{p}, y_{1}, \ldots, y_{q}\right)$, содер жащая точку $m$, $\varphi(m)=0$, и такие функиии $\left\{a_{i j}(y)\right\}_{i \in\{1, \ldots, q\}, j \in\{1, \ldots, r\}}, a_{i j}(0)=0$, что векторные กоภง

$$
\frac{\partial}{\partial x_{1}}, \quad \ldots, \quad \frac{\partial}{\partial x_{p}}, \quad Y_{1}=\sum_{i=1}^{q} a_{i 1}(y) \frac{\partial}{\partial y_{i}}, \quad \ldots, \quad Y_{r}=\sum_{i=1}^{q} a_{i r}(y) \frac{\partial}{\partial y_{i}}
$$

порождают расслоение векторов, касающихся слоев в окрестности $U$.

Это описание обеспечивает гомеоморфизм разбиения на $U$ с помощью локальных координат $\varphi$ на декартово произведение слоений $\mathscr{J} \times \mathscr{K}$ в $\mathbb{R}^{p} \times \mathbb{R}^{q}$, где у слоения $\mathscr{K}$ пространства $\mathbb{R}^{q}$ точка $\{0\}$ является 0-мерным слоем.

Разобъем многообразие $M$ на подмножества точек слоев одинаковой размерности. Связные компоненты этих множеств, назьваемые стратами, образуют стратификаuию многообразия $M$, которую мы обозначим через $\mathrm{S}_{\mathscr{F}}$. Если стратификация состоит из одной страты $\{M\}$, то слоение является регулярным. Гладкое отображение $f:(M, \mathscr{F})$ $\rightarrow\left(M^{\prime}, \mathscr{F}^{\prime}\right)$ многообразий назьвается отображсением слоений, если оно отображает слои в слои. Если $f$ является локальным диффеоморфизмом на образ, то он сохраняет размерность слоев и поэтому отображает страты многообразия $M$ в страты многообразия $M^{\prime}$. Группа диффеоморфизмов слоений будет обозначаться через $\operatorname{Diff}(M, \mathscr{F})$.

2.1.1. ПРИМЕРЫ.

(а) В любом открытом подмножестве $U \subset M$ индуцируется сингулярное слоение

$$
\mathscr{F}_{U}=\{\text { связные компоненты множества } L \cap U \mid L \in \mathscr{F}\} .
$$

Ассоциированная стратификация получается как

$$
\mathrm{S}_{\mathscr{F}_{U}}=\left\{\text { связные компоненты множества } S \cap U \mid S \in \mathrm{S}_{\mathscr{F}}\right\} .
$$

(b) Скажем, что диффеоморфизм слоений вида

$$
\varphi:\left(\mathbb{R}^{m-n-1} \times \mathbb{R}^{n+1}, \mathscr{H} \times \mathscr{K}\right) \rightarrow(U, \mathscr{F} U)
$$

является картой слоения, моделированной слоением $\left(\mathbb{R}^{m-n-1} \times \mathbb{R}^{n+1}, \mathscr{H} \times \mathscr{K}\right)$. 
(c) Рассмотрим связное регулярное слоение $(N, \mathscr{K})$. На декартовом произведении $N \times M$ получается сингулярное слоение

$$
\mathscr{K} \times \mathscr{F}=\left\{L_{1} \times L_{2} \mid L_{1} \in \mathscr{K}, L_{2} \in \mathscr{F}\right\}
$$

Ассоциированная стратификация при этом равна

$$
\mathrm{S}_{\mathscr{K} \times \mathscr{F}}=\left\{N \times S \mid S \in \mathrm{S}_{\mathscr{F}}\right\}
$$

(d) Рассмотрим $n$-мерную сферу $\mathbb{S}^{n}$, снабженную некоторьм сингулярным слоением $\mathscr{G}$ без 0 -мерных слоев. Отождествим диск $D^{n+1}$ с конусом

$$
c \mathbb{S}^{n}=\mathbb{S}^{n} \times\left[0,1\left[/ \mathbb{S}^{n} \times\{0\}\right.\right.
$$

с помощью отображения $x \mapsto[x /\|x\|,\|x\|]$, где $[u, t]$ - общая точка конуса. Рассмотрим на диске $D^{n+1}$ сингулярное слоение

$$
c \mathscr{G}=\{F \times\{t\}: F \in \mathscr{G}, t \in] 0,1[\} \cup\{\vartheta\},
$$

где $\vartheta=[u, 0]-$ вершина конуса. Индуцированная стратификация образует множество

$$
\mathrm{S}_{c} \mathscr{G}=\{S \times] 0,1\left[\mid S \in \mathscr{S}_{\mathscr{G}}\right\} \cup\{\vartheta\}
$$

поскольку $\mathscr{G}$ не содержит 0-мерных слоев. По техническим причинам будем рассматривать также случай $n=-1$, когда $\mathbb{S}^{n}=\varnothing$, а $c \mathbb{S}^{n}=\{\vartheta\}$. Если не предполагается противное, то на диске $D^{n+1}=c \mathbb{S}^{n}$ будем рассматривать слоение $c \mathscr{G}$, когда на сфере берется слоение $\left(\mathbb{S}^{n}, \mathscr{G}\right)$.

2.2. Конические слоения. Страты сингулярного слоения не обязаны быть многообразиями, а их расположение может быть очень сложным. Чтобы обеспечить построение когомологий пересечения, от стратификации $\mathscr{S}_{\mathscr{F}}$ требуется определенная степень регулярности и коничности (см. [1] для случая стратифицированных псевдомногообразий). Это потребовало от нас следующего определения.

Говорят, что слоение $(M, \mathscr{F})$ является коническим, если для любой точки $x \in M$ существует карта слоения $(U, \varphi)$, моделированная слоением $\left(\mathbb{R}^{m-n-1} \times c \mathbb{S}^{n}, \mathscr{H} \times c \mathscr{G}\right)$, где $\varphi(x)=0,\left(\mathbb{R}^{m-n-1}, \mathscr{H}\right)$ - простое слоение, а $\left(\mathbb{S}^{n}, \mathscr{G}\right)$ - некоторое коническое слоение без 0-мерных слоев. Это определение дается с помощью индукции по размерности многообразия $M$. Скажем, что карта $(U, \varphi)$ является конической картой в точке $x$, а слоение $\left(\mathbb{S}^{n}, \mathscr{G}\right)$ является зацеплением точки $x$. Заметим, что если точка $x$ содержится в страте $S$, то $\varphi^{-1}(S \cap U)=\mathbb{R}^{m-n-1} \times\{\vartheta\}$.

Заметим, что каждая страта является подмногообразием, вложенным в многообразие $M$. Слой (соответственно страта) слоения $\mathscr{F}$ (соответственно $\mathrm{S}_{\mathscr{F}}$ ), содержащий точку $x$, отображается с помошњю $\varphi$ в слой слоения $\mathscr{H}$, содержащий 0 (соответственно $\left.\mathbb{R}^{m-n-1}\right)$.

Число $n+1$ не зависит от выбора карты и равно коразмерности страты. Это число не может равняться 1 , поскольку коническое слоение на сфере $\left(\mathbb{S}^{n}, \mathscr{G}\right)$ не имеет 0 -мерных слоев. 
Для многообразий с краем тоже определяется понятие конического слоения. В этом случае точки края имеют карты, которые моделируются слоением

$$
\left(\mathbb{R}^{m-n-2} \times\left[0,1\left[\times c \mathbb{S}^{n}, \mathscr{H} \times \mathscr{I} \times c \mathscr{G}\right)\right.\right.
$$

где слоение $\mathscr{I}$ состоит из отдельных точек полуинтервала $[0,1[$ Край $(\partial M, \mathscr{F}$ әм) тоже является коническим слоением.

Из приведенного локального описания вытекают некоторые важные факты о стратификации $\mathscr{S}_{\mathscr{F}}$. В компактном случае семейство страт конечно, а в общем случае локально конечно. Замькание страты $S \in \mathscr{S}_{\mathscr{F}}$ является объединением страт. Положим $S_{1} \preceq S_{2}$, если $S_{1}, S_{2} \in \mathscr{S}_{\mathscr{F}}$ и $S_{1} \subset \bar{S}_{2}$. Это отношение является отношением частичного порядка, и поэтому $\left(\mathrm{S}_{\mathscr{F}}, \preceq\right)$ является частично упорядоченным множеством.

Глубина стратификации, обозначаемая $S_{\mathscr{F}}, \operatorname{depth} \mathscr{S}_{\mathscr{F}}$, определяется как наибольшее число $i$, для которого существует последовательность страт $S_{0} \prec S_{1} \prec \cdots \prec S_{i}$. Так что depth $S_{\mathscr{F}}=0$ тогда и только тогда, когда слоение $\mathscr{F}$ регулярно. Для любого открытого подмножества $U \subset M$ имеем $\operatorname{depth} \mathscr{S}_{\mathscr{F}_{U}} \leqslant \operatorname{depth} \mathrm{S}_{\mathscr{F}}$, а также имеем неравенство $\operatorname{depth} \mathrm{S}_{\mathscr{G}}=\operatorname{depth} \mathscr{S}_{\mathscr{H} \times \mathscr{G}}<\operatorname{depth} \mathrm{S}_{\mathscr{H} \times c \mathscr{G}}$ (см. примеры выше).

Минимальные страты в точности являются замкнутыми стратами. По индукции можно проверить, что максимальные страты имеют размерность $m$. Они называются регулярными стратами, а остальные страты называются сингулярными. Объединение сингулярных страт обозначается через $\Sigma \mathscr{F}$. Поскольку коразмерность сингулярных страт не меньше 2 , возникает только одна регулярная страта $R$, которая всюду плотна.

Чтобы продемонстрировать важность изучения гомологических и когомологических свойств сингулярных конических слоений, приведем две математические ситуации, в которых они появляются.

Гладкие действия компактных групп Ли (см. [16]). Для любого гладкого действия компактной групшы Ли $G$ на гладком многообразии $M$ естественная проекция $\pi_{G}: M \rightarrow$ $M / G$ на пространство орбит $M / G$ образует сингулярное коническое расслоение.

Интегрируемые гамильтоновы системы. Нгуен Тьен Зунг в своей недавней работе [17] рассмотрел следующую ситуацию. Рассмотрим гладкое гамильтоново отображение момента

$$
F=\left(F_{1}, \ldots, F_{n}\right):\left(M^{2 n}, \omega\right) \rightarrow \mathbb{R}^{n}
$$

вполне интегрируемой гамильтоновой системы с $n$ степенями свободы. Если $F-$ собственное отображение, что является достаточно естественным предположением, то связные компоненты многообразия уровня задают сингулярное слоение с компактными слоями. На регулярной части эти слои являются лагранжевыми торами $\mathbb{T}^{n}$ в многообразии $\left(M^{2 n}, \omega\right)$. Пусть $\mathscr{O}$ - пространство слоев этого слоения с факторной топологией. Тогда в случае интегрируемых систем общего положения пространство $\mathscr{O}$ является стратифицированным $n$-мерным многообразием, а естественная проекция $\pi: M \rightarrow \mathscr{O}$ является сингулярньм лагранжевым расслоением интегрируемой системы. Такие расслоения задаются действием Пуассона с помошью $n$ коммутируюших (по отношению к скобке Пуассона) функционально независимых функций на многообразии $M^{2 n}$. Было бы полезно сравнить наше определение конического расслоения с условием (Н2) в работе Нгуен Тьен Зунга. 
Для определения искаженных форм в следующем разделе мы будем использовать совместимость между картами слоения, сформулированную в следующей лемме, доказательство которой можно найти в [7].

Лемма 1. Рассмотрим две картыл слоения $\left(U_{1}, \varphi_{1}\right),\left(U_{2}, \varphi_{2}\right)$ в точке $x \in M$, причем $U_{1} \subset U_{2}$. Существует единственное вложсение слоений

$\varphi_{1,2}:\left(\mathbb{R}^{m-n-1} \times \mathbb{S}^{n} \times\left[0,1\left[, \mathscr{H}_{1} \times \mathscr{G}_{1} \times \mathscr{I}\right) \rightarrow\left(\mathbb{R}^{m-n-1} \times \mathbb{S}^{n} \times\left[0,1\left[, \mathscr{H}_{2} \times \mathscr{G}_{2} \times \mathscr{I}\right)\right.\right.\right.\right.$

для которого $P \circ \varphi_{1,2}=\varphi_{2}^{-1} \circ \varphi_{1} \circ P$, где $P: \mathbb{R}^{m-n-1} \times \mathbb{S}^{n} \times\left[0,1\left[\rightarrow \mathbb{R}^{m-n-1} \times c \mathbb{S}^{n}\right.\right.$ задется формулой $P(u, \theta, t)=(u,[\theta, t])$.

Из этого результата следует также, что зацепление $\left(\mathbb{S}^{n}, \mathscr{G}\right)$ в точке $x$ одинаково для всех точек страты $S$, содержащей $x$. Поэтому мы будем говорить, что $\left(\mathbb{S}^{n}, \mathscr{G}\right)$ является зацеплением страты $S$.

2.3. Частичное раздутие. Полезньм инструментом при проведении индуктивных рассуждений служит десингуляризация. Фиксируем некоторое коническое слоение $(M, \mathscr{F})$ строго положительной глубины. Под частичным раздутием мы будем понимать коническое слоение на многообразии с краем $(\widehat{M}, \widehat{\mathscr{F}})$ и некотороегладкоеотображение слоений $\mathscr{L}_{M}:(\widehat{M}, \widehat{\mathscr{F}}) \rightarrow(M, \mathscr{F})$, для которых вьполнены следуюшие условия:

1) ограничение $\mathscr{L}: \widehat{M}-\partial \widehat{M} \rightarrow M-S_{\min }$ является диффеоморфизмом, где $S_{\min }$ есть объединение замкнутых (минимальных) страт;

$2)$ для любой точки $x \in S_{\min }$ коммутируют отображения $\mathscr{L} \circ \widehat{\varphi}=\varphi \circ P$, где $(U, \varphi)-$ коническая карта точки $x$, а $\widehat{\varphi}:\left(\mathbb{R}^{m-n-1} \times \mathbb{S}^{n} \times[0,1[, \mathscr{H} \times \mathscr{G} \times \mathscr{I}) \rightarrow(\widehat{M}, \widehat{\mathscr{F}})\right.$ является вложением слоений.

Заметим, что каждое ограничение $\mathscr{L}: \mathscr{L}^{-1}(S) \rightarrow S$ на замкнутую страту $S$ является расслоением, допускаюшим атлас карт слоений и структурную групп, равную $\operatorname{Diff}\left(\mathbb{S}^{n}, \mathscr{G}\right)$ по отношению к некоторому коническому слоению $\left(\mathbb{S}^{n}, \mathscr{G}\right)$ на сфере.

ПРЕДЛОЖЕНИЕ 1. Частичное раздутие всегда существует и единственно. Справедливы также следующие утверждения:

3) $\operatorname{depth} \mathrm{S}_{\widehat{F}}=\operatorname{depth} \mathrm{S}_{\mathscr{F}}-1$;

4) $($ Ә $\widehat{M}, \widehat{\mathscr{F}}$ ә $\widehat{M})$ является коническим слоением.

Доказательство предложения 1 можно найти в [7].

\section{3. Базовые когомологии пересечения}

Имеется два способа определять искаженные формы: первьй использует систему трубчатых окрестностей (см. [4]), а второй использует глобальные раздутия (см. [5]). При изучении конических слоений мы предпочли вводить формы пересечения промежуточным путем: итерируя локальные раздутия многообразия $M$, мы получаем многообразие с границами $\widetilde{M}$, где слоение становится регулярным. Похожая процедура была развита в работе [5]. Некоторые дифференшиальные формы, заданные на регулярной части многообразия $M$, могут быть продолжены на многообразие $\widetilde{M},-$ они суть искаженные формы. Мы вводим это понятие внутренним образом, не приводя конструкции многообразия $\widetilde{M}$. 
Мы намерены работать с дифференциальньми формами на декартовом произведении $M \times\left[0,1\left[{ }^{p}\right.\right.$, где $M$ есть многообразие, а формы суть ограничения диффернциальных форм, заданных на большем пространстве $M \times]-1,1\left[{ }^{p}\right.$.

3.1. Искаженные формы. Комплекс $\Pi^{*}\left(M \times\left[0,1\left[^{p}\right)\right.\right.$ искажсенных форм вводится индукцией по глубине стратификации $\operatorname{depth} S_{\mathscr{F}}$. Если глубина равна 0, то полагаем $\Pi^{*}\left(M \times\left[0,1\left[^{p}\right)=\Omega^{*}\left(M \times\left[0,1\left[^{p}\right)\right.\right.\right.\right.$. В общем случае рассмотрим дифференшиальную форму $\omega \in \Omega^{*}\left(\left(M-\Sigma_{\mathscr{F}}\right) \times\left[0,1\left[^{p}\right)\right.\right.$. Поскольку в конической карте $(U, \varphi)$ регулярная часть пространства $\mathbb{R}^{m-n-1} \times c \mathbb{S}^{n}$ в точности совпадает с $\left.\mathbb{R}^{m-n-1} \times\left(\mathbb{S}^{n}-\Sigma_{\mathscr{G}}\right) \times\right] 0,1[$, то

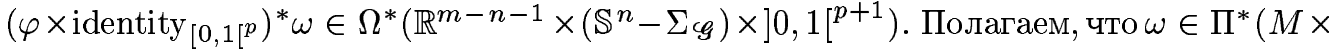
$\left[0,1\left[^{p}\right)\right.$, если для любой конической карты $(U, \varphi)$ форма

$$
\begin{gathered}
\left(\varphi \times \text { identity }_{\left[0,1\left[^{p}\right)^{*} \omega\right.}\right. \text { продолжается до формы } \\
\omega_{\varphi} \in \Pi^{*}\left(\mathbb{R}^{m-n-1} \times \mathbb{S}^{n} \times\left[0,1\left[\left[^{p+1}\right) .\right.\right.\right.
\end{gathered}
$$

Построенное пространство $\Pi^{*}\left(M \times\left[0,1\left[^{p}\right)\right.\right.$ является дифференциальной градуированной коммутативной алгеброй (DGCA для краткости), поскольку $(\omega+\eta)_{\varphi}=\omega_{\varphi}+\eta_{\varphi}$, $(\omega \wedge \eta)_{\varphi}=\omega_{\varphi} \wedge \eta_{\varphi}$ и $(d \omega)_{\varphi}=d \omega_{\varphi}$

3.1.1. СвоЙСтва (см. [7]).

(а) Пусть $(N, \mathscr{H})$ - регулярное слоение. Частичным раздутием слоения $N \times c \mathbb{S}^{n}$ является слоение $N \times \mathbb{S}^{n} \times[0,1[$ с естественной проекцией $(x, \theta, t) \mapsto(x,[\theta, t])$. Здесь появляется сомножитель [0, 1[. Десингуляризация добавляет дополнительные сомножители $[0,1[$.

(b) Рассмотрим частичное раздутие $\mathscr{L}_{M}: \widehat{M} \rightarrow M$ конического слоения. Любая искаженная форма $\omega$ на слоении $M$ определяет искаженную форму $\widehat{\omega}$ на $\widehat{M}$, продолжающую форму $\mathscr{L}_{M}^{*} \omega$. Это отображение $\omega \mapsto \widehat{\omega}$ задает изоморфизм между пространствами $\Pi^{*}(M)$ и $\Pi^{*}(\widehat{M})$.

(c) Для любой искаженной формы и любой конической карты вьполняется условие (1).

(d) Существуют дифференциальные формы на $M-\Sigma_{\mathscr{F}}$, которые не являются искаженньми. Любая форма $\omega$, определенная на всем многообразии $M$ является искаженной.

(е) Рассмотрим открытоепокрытие $\{U, V\}$ на $M$. Существует гладкое разбиение единишы на многообразии $M$, подчиненное этому покрытию. Наличие такого разбиения единишы влечет точность короткой последовательности Майера-Виеториса

$$
0 \rightarrow \boldsymbol{\Pi}^{*}(M) \rightarrow \boldsymbol{\Pi}^{*}(U) \oplus \boldsymbol{\Pi}^{*}(V) \rightarrow \boldsymbol{\Pi}^{*}(U \cap V) \rightarrow 0 .
$$

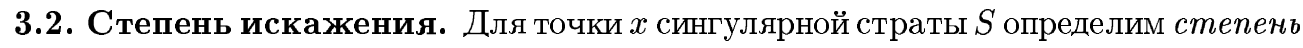
искажсения $\|\omega\|_{x}$ как наименьшее число $k$, для которого вьполнено условие

$$
i_{\zeta_{0}} \cdots i_{\zeta_{k}} \omega_{\varphi} \equiv 0
$$

для каждой конической карты $(U, \varphi)$ точки $x$, где $\varphi^{-1}(x)=(a, \vartheta)$, и для каждого семейства векторных полей $\left\{\zeta_{i}\right\}_{i=0}^{k}$ на $\{a\} \times\left(\mathbb{S}^{n}-\Sigma_{\mathscr{G}}\right) \times\{0\}$. Здесь $i$ с индексом обозначает внутреннее произведение. Если форма $\omega_{\varphi}$ обращается в нуль на $\{a\} \times\left(\mathbb{S}^{n}-\Sigma_{\mathscr{G}}\right) \times\{0\}$, мы будем писать $\|\omega\|_{x}=-\infty$. Определим степень искажения $\|\omega\|_{S}$ по формуле

$$
\|\omega\|_{S}=\sup \left\{\|\omega\|_{x} \mid x \in S\right\} .
$$


3.2.1. СвойствА. Напомним некоторые основные свойства искаженных форм (см. [7]).

(a) Степень искажения $\|\omega\|_{x}$ не зависит от выбора конической карты точки $x$.

ДокаЗАТЕЛЬСтво. Рассмотрим две карты слоения $\left(U_{1}, \varphi_{1}\right)$ и $\left(U_{2}, \varphi_{2}\right)$ точки $x$ с условием $U_{1} \subset U_{2}$. Имеем $\varphi_{1}^{-1}(x)=\left(a_{1}, \vartheta\right)$ и $\varphi_{2}^{-1}(x)=\left(a_{2}, \vartheta\right)$. Из леммы 2.2 .1 следует, что $\omega_{\varphi_{1}}=\Phi_{1,2}^{*} \omega_{\varphi_{2}}$, а ограничение $\Phi_{1,2}:\left\{a_{1}\right\} \times\left(\mathbb{S}^{n}-\Sigma \mathscr{G}_{1}\right) \times\{0\} \rightarrow\left\{a_{2}\right\} \times\left(\mathbb{S}^{n}-\Sigma \mathscr{G}_{2}\right) \times\{0\}$ является диффеоморфизмом. Получаем, что $\|\omega\|_{x}^{\varphi_{1}}=\|\omega\|_{x}^{\varphi_{2}}$.

(b) Для двух искаженных форм $\omega$ и $\eta$ и сингулярной страты $S$ имеем

$$
\|\omega+\eta\|_{S} \leqslant \max \left\{\|\omega\|_{S},\|\eta\|_{S}\right\}, \quad\|\omega \wedge \eta\|_{S} \leqslant\|\omega\|_{S}+\|\eta\|_{S} .
$$

(c) Степень искажения искаженной функции равна 0 или $-\infty$. Степень искажения формы $\omega$ на $M$ равна 0 или $-\infty$.

(d) Пусть $(N, \mathscr{H})$ - регулярное слоение, а $a_{0} \in N$ - отмеченная точка. Обозначим через pr: $N \times M \rightarrow M$ каноническую проекцию, а через $\iota: M \rightarrow N \times M$ вложение, определенное по формуле $\iota(x)=\left(a_{0}, x\right)$. Тогда для любых $\omega \in \boldsymbol{\Pi}^{*}(M)$ и $\eta \in \boldsymbol{\Pi}^{*}(N \times M)$ имеют место неравенства $\left\|\mathrm{pr}^{*} \omega\right\|_{N \times S} \leqslant\|\omega\|_{S}$ и $\left\|\iota^{*} \omega\right\|_{S} \leqslant\|\omega\|_{N \times S}$ для каждой сингулярной страты многообразия $M$.

(е) Рассмотрим искаженную форму $\omega \in \boldsymbol{\Pi}^{*}\left(c^{n}\right)$. Ее степень искажения относительно вершины равна

$$
\|\omega\|_{\{\vartheta\}}= \begin{cases}-\infty, & \text { если } \omega_{\varphi} \equiv 0 \text { на } \mathbb{S}^{n} \times\{0\} \\ \operatorname{deg} \omega & \text { в противном случае }\end{cases}
$$

где $(U, \varphi)$ - произвольная коническая карта точки $\{\vartheta\}$.

(f) Искаженная форма, для которой $\|\omega\|_{S} \leqslant 0$ и $\|d \omega\|_{S} \leqslant 0$, индуцирует дифференциальную форму $\omega_{S}$ на $S$. Если это условие вьполняется для каждой страты, то $\omega \equiv\left\{\omega_{S}\right\}$ является контролируемой формой в смысле Верона (см. [6]).

3.3. Базовые когомологии. Рассмотрим слоение $(M, \mathscr{F})$. Дифференциальная форма $\omega \in \Omega^{*}(M)$ называется базовой, если $i_{X} \omega=i_{X} d \omega=0$ для каждого векторного поля $X$ на многообразии $M$, касаюшегося слоения. Через $\Omega^{*}(M / \mathscr{F})$ обозначим комплекс базовых форм. Поскольку сумма и произведение базовых форм тоже являются базовыми, этот комплекс образует DGCA-алгебру. Ее когомологии $H^{*}(M / \mathscr{F})$ назьваются базовыми когомологиями слоения $(M, \mathscr{F})$, которые тоже образуют DGCA-алгебру. Если $F \subset M$ - насьшенное замкнутое подмножество, то мы будем обозначать через $\Omega^{*}((M, F) / \mathscr{F})$ подкомплекс базовых форм на $M$, обращаюшихся в нуль на $F$. Когомологии этого подкомплекса обозначим через $H^{*}((M, F) / \mathscr{F})$.

3.3.1. СВОЙСТВА.

(а) Гладкое отображение слоений $f:\left(M_{1}, \mathscr{F}_{1}\right) \rightarrow\left(M_{2}, \mathscr{F}_{2}\right)$ индуцирует гомоморфизм DGCA-адгебр $f^{*}: \Omega^{*}\left(M_{2} / \mathscr{F}_{2}\right) \rightarrow \Omega^{*}\left(M_{1} / \mathscr{F}_{1}\right)$.

(b) Открытое покрытие $\{U, V\}$ многообразия $M$ с помощью насышенных открытых множеств называется базовым покрытием, если существует подчиненное ему разбиение единишы, построенное при помощи базовых функций. Для базового покрытия имеет место точная короткая последовательность Майера-Виеториса

$$
0 \rightarrow \Omega^{*}(M / \mathscr{F}) \rightarrow \Omega^{*}\left(U / \mathscr{F}_{U}\right) \oplus \Omega^{*}\left(V / \mathscr{F}_{V}\right) \rightarrow \Omega^{*}\left((U \cap V) / \mathscr{F}_{U \cap V}\right) \rightarrow 0
$$


где отображения задаются по правилу $\omega \mapsto(\omega, \omega)$ и $(\alpha, \beta) \mapsto \alpha-\beta$. Для произвольных покрытий указанная последовательность, вообще говоря, не точна.

3.4. Базовые когомологии пересечения. Рассмотрим коническое слоение $(M, \mathscr{F}) . \quad$ Функиией искажения называется отображение $\bar{p}: \mathrm{S}_{\mathscr{F}} \rightarrow \mathbb{Z}$. Нулевое искажсение $\overline{0}$ задается равенством $\overline{0}(S)=0$. С гладким отображением слоений $f:\left(M^{\prime}, \mathscr{F}^{\prime}\right) \rightarrow(M, \mathscr{F})$ (соответственно $\left.f:(M, \mathscr{F}) \rightarrow\left(M^{\prime}, \mathscr{F}^{\prime}\right)\right)$ ассоциируется искажение на слоении $\left(M^{\prime}, \mathscr{F}^{\prime}\right)$, также обозначаемое через $\bar{p}$ и задаваемое равенством $\bar{p}\left(S^{\prime}\right)=\bar{p}(S)$, где $S^{\prime} \in \mathrm{S}_{\mathscr{F}}$ и $S \in \mathrm{S}_{\mathscr{F}}$ такие, что $f\left(S^{\prime}\right) \subset S$ (соответственно $\left.S=f^{-1}\left(S^{\prime}\right)\right)$.

Базовые когомологии пересечения возникают при рассмотрении базовых форм, чья степень искажения контролируется с помощью функции искажения. Будем обозначать через

$$
\Omega_{\bar{p}}^{*}(M / \mathscr{F})=\left\{\omega \in \Omega^{*}\left(\left(M-\Sigma_{\mathscr{F}}\right) / \mathscr{F}_{M-\Sigma}\right) \mid \max \left(\|\omega\|_{S},\|d \omega\|_{S}\right) \leqslant \bar{p}(S) \forall S \in \mathrm{S}_{\mathscr{F}}\right\}
$$

комплекс базовых форм, степень искажения которых (а также их дифференциалов) ограничена искажением $\bar{p}$. Когомологии $\mathbb{H}_{\bar{p}}^{*}(M / \mathscr{F})$ этого комплекса назьваются базовыми когомологиями пересечения многообразия $M$, или для краткости БКП, относительно функции искажения $\bar{p}$.

3.4.1. СвоЙСТВА.

(а) Вложение слоений $f:\left(M_{1}, \mathscr{F}_{1}\right) \rightarrow\left(M_{2}, \mathscr{F}_{2}\right)$ индуцирует гомоморфизм DGCA-алгебр $f^{*}: \Omega_{\bar{p}}^{*}\left(M_{2} / \mathscr{F}_{2}\right) \rightarrow \Omega_{\bar{p}}^{*}\left(M_{1} / \mathscr{F}_{2}\right)($ см. 3.3.1(a) и $3.2 .1(\mathrm{~b}))$.

(b) Рассмотрим базовое покрытие $\{U, V\}$ многообразия $M$. Короткая последовательность Майера-Виеториса

$$
0 \rightarrow \Omega_{\bar{p}}^{*}(M / \mathscr{F}) \rightarrow \Omega_{\bar{p}}^{*}\left(U / \mathscr{F}_{U}\right) \oplus \Omega_{\bar{p}}^{*}\left(V / \mathscr{F}_{V}\right) \rightarrow \Omega_{\bar{p}}^{*}\left((U \cap V) / \mathscr{F}_{U \cap V}\right) \rightarrow 0
$$

точна. Для произвольных покрытий этот результат не имеет места.

Базовые когомологии пересечения совпадают с базовыми когомологиями, если слоение $\mathscr{F}$ регулярно, поскольку тогда оно состоит из одной страты. Но они также обобщают когомологии пересечения в смысле Горески-МакФерсона (см. [1]) в случае, когда пространство слоев $B$ принадлежит правильной категории стратифицированных псевдомногообразий (см. теорему 1).

ПРЕДЛОЖЕНИЕ 2. Пусть $\left(\mathbb{R}^{k}, \mathscr{H}\right)$ - простое слоение, а $(M, \mathscr{F})$ - коническое слоение. Для произвольной функиии искажения $\bar{p}$ проекция

$$
\operatorname{pr}: \mathbb{R}^{k} \times\left(M-\Sigma_{\mathscr{F}}\right) \rightarrow\left(M-\Sigma_{\mathscr{F}}\right)
$$

индуиирует изоморфизм $\mathbb{H}_{p}^{*}\left(\mathbb{R}^{k} \times M / \mathscr{H} \times \mathscr{F}\right) \cong \mathbb{H}_{p}^{*}(M / \mathscr{F})$.

ПРЕДЛОЖЕНИЕ 3. Пусть $\mathscr{G}$ - коническое слоение на сфере $\mathbb{S}^{n}$. Для произвольной функиии искажения $\bar{p}$ проекиия $\left.\mathrm{pr}:\left(\mathbb{S}^{n}-\Sigma_{\mathscr{G}}\right) \times\right] 0,1\left[\rightarrow\left(\mathbb{S}^{n}-\Sigma_{\mathscr{G}}\right)\right.$ индуиирует изоморфизм

$$
\mathbb{H} \mathbb{L}_{\bar{p}}^{i}\left(c S^{k} / c^{\mathscr{G}}\right)= \begin{cases}\mathbb{H}_{p}^{i}\left(S^{k} / \mathscr{G}\right), & \text { если } i \leqslant \bar{p}(\{\vartheta\}), \\ 0, & \text { если } i>\bar{p}(\{\vartheta\}) .\end{cases}
$$

Этот результат показывает, что базовые когомологии пересечения не определяются когомологиями многообразия $M$. 


\section{4. Стратифицированные псевдомногообразия}

Стратифииированное псевдомногообразие $X$ задается (см. [1])

- паракомпактным пространством $X$;

- локально конечньм разбиением $\mathrm{S}_{X}$, назьваемым стратификаиией, состоящим из связных гладких многообразий, назьваемых стратами.

Требуется, чтобы у каждой точки пространства $X$ была окрестность, гомеоморфная локальной модели $\mathbb{R}^{p} \times c L$, причем гомеоморфизмы отображали страты в страты, и существовала плотная страта $R$, назьваемая регулярной стратой.

Инициированные Горески и МакФерсоном результаты показали, что для изучения такого сорта сингулярных пространств наиболее подходящими оказались когомологии пересечения (см. [1] , а также [3], [2]). Главньй результат настоящего исследования заключается в том, что когомологии пересечения пространства $B$ изоморфны базисньц когомологиям пересечения слоения $\mathscr{F}$.

4.1. Отсутствие голономии. В этом пункте рассмотрим случай, когда коническое слоение $\mathscr{F}$ имеет собственные слои без голономии. Через $B$ обозначим пространство слоев, а через $\pi: M \rightarrow B$ каноническую проекцию. Скажем, что коническое слоение $(M, \mathscr{F})$ является сингулярным расслоением, если для каждой сингулярной страты $S$ стратификации $S_{\mathscr{F}}$ имеют место следуюшие свойства:

а) ограничение $\pi: S \rightarrow \pi(S)$ является гладким расслоением (условие голономии $\partial$ 几я $\left.\mathscr{F}_{S}\right)$;

b) зацепление $\left(\mathbb{S}^{n}, \mathscr{G}\right)$ страты $S$ является сингулярным расслоением (индуктивное условие);

с) след слоя $\mathscr{F}$ на $\mathbb{S}^{n}$ связен (условие голономии для страты $S$ на многообразии $M)$.

Для такого вида слоений получается следующий результат.

ПРЕДЛОЖЕНИЕ 4. Пространство слоев сингулярного расслоения является стратифицированным псевдомногообразием.

ДокАЗАТЕЛЬСТво. Пусть $(M, \mathscr{F})$ - сингулярное расслоение, а $B$ - пространство слоев. Поскольку многообразие $M$ имеет счетный базис хороших конических карт $\{(\varphi, U)\}$, то пространство $B$ имеет счетный базис, составленный из карт $\{(\psi, \pi(U)\}$. Значит, поскольку $B$ регулярно, то оно паракомпактно. Рассмотрим стратификацию $\mathrm{S}_{B}=\left\{\pi(S) \mid S \in \mathrm{S}_{\mathscr{F}}\right\}$, что следует из а) и локальной конечности $\mathscr{S}_{\mathscr{F}}$. Проведем индукцию по глубине стратификации. На первом шаге получаем обычную теорему де Рама.

В общем случае рассмотрим коническую карту $(\varphi, U)$ страты $S$. Поскольку слоение $\left(S, \mathscr{F}_{S}\right)$ является расслоением, любая точка страты $S$ допускает карту, моделируемую с помощью слоения $\left(\mathbb{R}^{m-n-1}, \mathscr{H}\right) \equiv\left(\mathbb{R}^{r}, \mathscr{K}\right) \times\left(\mathbb{R}^{s}, \mathscr{I}\right)$, где $\mathscr{K}$ есть слоение с одним слоем, а $\mathscr{I}$ есть слоение, состоящее из отдельньх точек. Скажем, что карта $(\varphi, U)$ является хорошей конической картой, если она моделируется с помошю $\left(\mathbb{R}^{r} \times \mathbb{R}^{s} \times c \mathbb{S}^{n}, \mathscr{K} \times \mathscr{I} \times c \mathscr{G}\right)$.

Хорошая коническая карта $\varphi$ порождает стратифицированное вложение $\psi: \mathbb{R}^{s} \times$ $c \pi_{\mathbb{S}^{n}}\left(\mathbb{S}^{n}\right) \rightarrow B\left(\right.$ см. с)), где $\pi_{\mathbb{S}^{n}}$ есть каноническая проекция на $\mathbb{S}^{n}$. Здесь мы можем применить индукцию, поскольку $\left(\mathbb{S}^{n}, \mathscr{G}\right)$ является сингулярным расслоением (см. b) ). 
Применяя ту же технику, можно непосредственно показать, что существет регулярная страта $R$.

Гомологии пересечения в смысле Горески-Макферсона $\mathbb{H}_{p}^{*}(B)$ были ими введены для того, чтобы изучать стратифишированные псевдомногообразия (см. [1], а также [3], [2]). Главный результат настоящей работы заключается в установлении связи между этими гомологиями пересечений и базисньми когомологиями пересечений слоения $\mathscr{F}$. Напомним, что две функции искажения $\bar{p}$ и $\bar{q}$ на пространстве $B$ двойственны, если

$$
\bar{q}(\pi(S))+\bar{p}(\pi(S))=\operatorname{codim}_{B}(\pi(S))-2
$$

для каждой сингулярной страты $\pi(S)$. Заметим, что функция искажения $\bar{p}$ определяет искажение на $B$ равенством $\bar{p}(\pi(S))=\bar{p}(S)$.

ТеОРема 1. Пусть $(M, \mathscr{F})$ - сингулярное расслоение и $B$ - пространство орбит. Тогда

$$
\mathbb{H}_{\bar{q}}^{*}(M / \mathscr{F}) \cong \mathbb{H}_{\bar{p}}^{*}(B),
$$

где $\bar{p} и \bar{q}-$ двойственные функиии искажения.

Доказательство изложено в разделе 5 .

\section{2. Наличие голономий.}

4.2.1. ПримеР. Рассмотрим сферу $\mathbb{S}^{3}$, снабженную римановым слоением $\mathscr{H}$, которое задается действием Хопфа, т.е. $z \cdot\left(u_{1}, u_{2}\right)=\left(z \cdot u_{1}, z \cdot u_{2}\right)$. С другой стороны, рассмотрим пространство $\mathbb{R}^{6}=c \mathbb{S}^{5}$, снабженное сингулярньм римановьг слоением $c \mathscr{Q}$, которое задается действием тора $\mathbb{T}^{2} \times c \mathbb{S}^{5} \rightarrow c \mathbb{S}^{5}$ по формуле

$$
\left(z_{1}, z_{2}\right) \cdot\left[\left(v_{1}, v_{2}, v_{3}\right), t\right]=\left[\left(z_{1} \cdot v_{1}, z_{1} z_{2} \cdot v_{2}, z_{1} z_{2}^{2} \cdot v_{3}\right), t\right]
$$

Имеется три типа слоев: точки, окружности и торы. Имеется три типа страт: точка (чье зацепление есть $\left.\left(\mathbb{S}^{5}, \mathscr{Q}\right)\right)$, три цилиндра (чьи зацепления суть $\left(\mathbb{S}^{3}, \mathscr{H}\right)$ ) и регулярная страта. В самом деле, сингулярная часть есть букет $\mathbb{D}^{2} \vee \mathbb{D}^{2} \vee \mathbb{D}^{2}$, снабженньй слоением, порожденньм естественным действием окружности $\mathbb{S}^{1}$ на каждом диске. Конечная группа $\mathbb{Z}_{3}$ свободно действует на произведении $\mathbb{S}^{3} \times c \mathbb{S}^{5}$ по формуле

$$
e^{2 \pi i / 3} \cdot\left(\left(u_{1}, u_{2}\right),\left[\left(u_{1}, u_{2}, u_{3}\right), t\right]\right)=\left(\left(u_{1}, e^{2 \pi i / 3} \cdot u_{2}\right),\left[\left(u_{3}, u_{1}, u_{2}\right), t\right]\right) .
$$

На этом произведении имеется три типа слоев: окружности, торы и трехмерные торы - и три типа страт: $\mathbb{S}^{3}$, три копии пространства $\mathbb{S}^{3} \times \mathbb{S}^{1} \times 10,1[$ и регулярная страта. Заметим, что действие группы $\mathbb{Z}_{3}$ переставляет три 4-мерных страты. Поскольку это действие сохраняет слоение $\mathscr{H} \times c \mathscr{Q}$, на фактормногообразии $M=\mathbb{S}^{3} \times \mathbb{Z}_{3} c \mathbb{S}^{5}$ получается сингулярное риманово слоение $\mathscr{F}$. На нем тоже имеется три типа слоев: окружности, торы и трехмерньне торы - и три типа страт. Минимальная страта $S$ есть линзовое пространство $L(1,3)=\mathbb{S}^{3} / \mathbb{Z}^{3}$, у которого зацепление равно $\left(L_{S}, \mathscr{G}\right)=\left(\mathbb{S}^{5}, \mathscr{Q}\right)$. Имеется одна 4 -мерная страта, произведение $\left.\mathbb{S}^{3} \times \mathbb{S}^{1} \times\right] 0,1[$, у которой зацепление равно $\left(L_{S}, \mathscr{G}\right)=\left(\mathbb{S}^{3}, \mathscr{H}\right)$. Последняя страта является регулярной. 
Обозначим через $B$ пространство слоев $M / \mathscr{F}$, а через $\pi: M \rightarrow B$ каноническую проекцию. Образ $\pi(S)=L(3,1) / \mathscr{F}=\mathbb{S}^{2} / \mathbb{Z}^{3}$ не является многообразием, а всего лишњ орбифолдом.

Здесь уже видно отличие от сингулярных расслоений. Страты не обязательно являются многообразиями, а только орбифолдами (см., например, [8]). Но имеется другое важное отличие, связанное с "зацеплениями". Естественная проекция $\tau: M \rightarrow S$ является "слоеной" трубчатой окрестностью пространства $S$ со слоем $c \mathbb{S}^{5}$.

Индуцированное отображение $\sigma: \pi(T) \rightarrow \pi(S)$ является трубчатой окрестностью пространства $\pi(S)$ со слоем факторпространство $\left(\mathbb{S}^{5} / \mathscr{G}\right) / \mathbb{Z}_{3}$. "Зацепление" $L_{\pi(S)}$ в $\pi(S)$ поэтому равно $\left(\mathbb{S}^{5} / \mathscr{Q}\right) / \mathbb{Z}_{3}$. Другими словами, имеет место не $L_{\pi(S)}=L_{S} / \mathscr{G}$, а $L_{\pi(S)}=$ $\left(L_{S} / \mathscr{G}\right) / \mathbb{Z}^{3}$. Можно показать, что след слоения $\mathscr{F}$ на зацеплении $S$ в точности суть орбиты $\mathbb{Z}^{3} \cdot \mathscr{Q}$.

4.2.2. Категория орбифолдов. Предыдущие рассмотрения приводят к следующим понятиям. Стратифицированный псевдоорбифолд определяется путем замены "многообразия" на "орбифолд", а “диффеоморфизма" на “изоморфизм орбифолдов". Другими словами, стратифицированный орбифолд задается с помошью

- паракомпактного пространства $X$;

- локально конечного подразбиения $\mathrm{S}_{X}$, назьваемого стратификаиией, составленного из связных гладких орбифолдов.

Каждая точка пространства $X$ должна иметь окрестность, гомеоморфную локальной модели $U \times c L$, и должна иметься плотная (регулярная) страта $R$.

Аналогично расширим понятие сингулярного расслоения. Скажем, что коническое слоение $(M, \mathscr{F})$ является сингулярным расслоением Зейферта, если для каждой сингулярной страты $S$ слоения $\mathrm{S}_{\mathscr{F}}$

a) ограничение $\pi: S \rightarrow \pi(S)$ является расслоением Зейферта;

b) зацепление $\left(\mathbb{S}^{n}, \mathscr{G}\right)$ страты $S$ является сингулярным расслоением Зейферта;

c) след слоения $\mathscr{F}$ на $\mathbb{S}^{n}$ задается орбитами $H \cdot \mathscr{G}$, где $H$ есть конечная подгруппа в групше $O(n+1, \mathscr{G})=O(n+1) \cap \operatorname{Diff}\left(\mathbb{S}^{n}, \mathscr{G}\right)$.

ПРЕДЛОЖЕНИЕ 5. Пространство сингулярного расслоения Зейферта является стратифииированным псевдоорбифолдом.

ДокАЗАТЕЛЬСтво. Пусть $(M, \mathscr{F})$ есть сингулярное расслоение, а $B$ - его пространство слоев. Из [8] известно, что элементы семейства $\mathrm{S}_{B}=\left\{\pi(S) \mid S \in \mathrm{S}_{\mathscr{F}}\right\}$ являются орбифолдами. Проводя рассуждения, аналогичные рассуждениям предложения 4 , получаем, что пространство $B$ паракомпактно, а семейство $\mathrm{S}_{B}$ локально конечно. На первом шаге получаем, что проекция $\pi$ является расслоением Зейферта, а пространство $B$ является стратифицированньм псевдоорбифолдом глубины 0 . В общем случае рассмотрим коническую карту $(\varphi, U)$ в страте $S$. Локальная модель для точки в образе $\pi(S)$ есть произведение $\left(\mathbb{R}^{p} / \Gamma, c\left(\mathbb{S}^{n} / \mathscr{G}\right) / H\right)$, где $\Gamma \subset O(p)$ есть конечная подгрупша, $\left(S^{n}, \mathscr{G}\right)$ есть зацепление в $S$ и $H \subset O(n+1, \mathscr{G})$ есть конечная подгруппа. По индукции получаем, что $\mathbb{S}^{n} / \mathscr{G}$, а, значит, и $\left(\mathbb{S}^{n} / \mathscr{G}\right) / H$ являются стратифицированными псевдоорбифолдами.

4.2.3. Сингулярные римановы слоения. Из [18] (см. также [7]) известно, что каждая страта $S$ сингулярного риманова слоения $(M, \mathscr{F})$ имеет трубчатую поверхность, 
назьвемую трубчатой окрестностью слоения, $\tau: T \rightarrow S$ с атласом карт слоения вида $\left\{\varphi:\left(\tau^{-1}(U), \mathscr{F}\right) \rightarrow\left(U \times c \mathbb{S}^{n}, \mathscr{F} \times c \mathscr{G}\right)\right\}$, чья структурная группа есть группа $O(n+1, \mathscr{F})=\{A \in O(n+1) \mid A$ сохраняет $\mathscr{G}\}$. Здесь $\left(\mathbb{S}^{n}, \mathscr{G}\right)$ обозначает зацепление в $S$. Сингулярное риманово слоение представляет пример стратифишированного псевдомногообразия.

ПРЕДЛОЖЕНИЕ 6. Пространство слоев сингуляного риманова слоения с компактными слоями является стратифицированным орбифолдом.

ДокАЗАТЕЛЬСтво. Пусть $(M, \mathscr{F})$ - сингулярное риманово слоение, $B$ - пространство слоев, а $\pi: M \rightarrow B$ - естественная проекция. Поскольку ограничение слоения $\mathscr{F}$ на страту $S$ является римановым слоением с компактньми слоями, то ограничение $\pi: S \rightarrow$ $\pi(S)$ является расслоением Зейферта (см. [8]). Таким образом, получается условие а).

Для доказательства условия b) заметим, что $\left(\mathbb{S}^{n}, \mathscr{G}\right)$ является сингулярным римановым слоением на многообразии, для которого $\operatorname{depth} \mathrm{S}_{\mathscr{G}}<\operatorname{depth} \mathrm{S}_{\mathscr{F}}$. Индукцией по глубине стратификации получаем условие b). На первом шаге индукции проекция $\pi$ в точности является расслоением Зейферта, а предложение превращается в теорему де Рама для орбифолдов.

Слоение $\mathscr{F}$ не всегда индуцирует на страте $\mathbb{S}^{n}$ слоение $\mathscr{G}$ (см. пример 4.2.1). Рассмотрим трубчатую окрестность $\tau: T \rightarrow S$ слоения со структурной группой $O(n+1, \mathscr{G})$. След слоения $\mathscr{F}$ на слое $\mathbb{S}^{n}$ задается орбитами $H \cdot \mathscr{G}$, где $H=\{A \in O(n+1, \mathscr{G})$, сохряняюших слои $\}$. Поскольку $H$ сохраняет регулярную страту, то по соображениям размерности получаем, что $\operatorname{dim} H=0$. Это доказьвает свойство с).

Так же, как и в случае тривиальной голономии, базисные когомологии пересечения обобщают когомологии пересечения пространства слоев.

Теорема 2. Пусть $(M, \mathscr{F})$ - сингулярное расслоение Зейферта, а В - пространство слоев. Тогда

$$
\mathbb{H}_{\bar{q}}^{*}(M / \mathscr{F}) \cong \mathbb{H}_{\bar{p}}^{*}(B),
$$

әде $\bar{p}$ и $\bar{q}$ - двойственные функиии искажения.

ДокАЗАТЕЛьСТво. Следуя доказательству теоремы 1 , задача сводится к локальной проблеме на пространстве $B$. Напомним, что локальной моделью для пространства $B$ служит $\left(\mathbb{R}^{p} / \Gamma, c \pi\left(\mathbb{S}^{n}\right) / H\right)$, где $\Gamma \subset O(p)$ является конечной подгруппой, $\left(S^{n}, \mathscr{G}\right)$ является зацеплением в $S, \pi: \mathbb{S}^{n} \rightarrow \pi\left(\mathbb{S}^{n}\right)$ обозначает каноническую проекцию, ассоциированную с $\mathscr{G}$, а $H \subset O(n+1, \mathscr{G})$ является конечной подгруппой. Стягивая первьй сомножитель, задачу сводим к утверждению

$$
H^{*}\left(\Omega_{\bar{q}}\left(\pi\left(\mathbb{S}^{n}\right) / H\right)\right) \cong H^{*}\left(\operatorname{Hom}\left(S C_{\bar{p}}\left(\pi\left(\mathbb{S}^{n}\right) / H\right), \mathbb{R}\right)\right)
$$

Поскольку $\mathbb{S}^{n} / \mathscr{G}$ является стратифицированньм псевдоорбифолдом, по индукции получаем

$$
H^{*}\left(\Omega_{\bar{q}}\left(\pi\left(\mathbb{S}^{n}\right)\right)\right) \cong H^{*}\left(\operatorname{Hom}\left(S C_{\bar{p}}\left(\pi\left(\mathbb{S}^{n}\right)\right), \mathbb{R}\right)\right) .
$$

Используя конечность групшы $H$ (см. [19]), получаем изоморфизмы с подпространствами неподвижных точек

$$
H^{*}\left(\Omega_{\bar{q}}\left(\pi\left(\mathbb{S}^{n}\right) / H\right)\right) \cong\left(H^{*}\left(\Omega_{\bar{q}}\left(\pi\left(\mathbb{S}^{n}\right)\right)\right)\right)^{H}
$$


и

$$
H^{*}\left(\operatorname{Hom}\left(S C_{\bar{p}}\left(\pi\left(\mathbb{S}^{n}\right) / H\right), \mathbb{R}\right)\right) \cong\left(H^{*}\left(\operatorname{Hom}\left(S C_{\bar{p}}\left(\pi\left(\mathbb{S}^{n}\right)\right), \mathbb{R}\right)\right)\right)^{H}
$$

Для завершения доказательства достаточно заметить естественность изоморфизма (2).

Рассмотрим коническое слоение с компактньпи слоями $\mathscr{F}$ на $M$, которое мы будем назьвать компактным коническим слоением. Это слоение индуцирует на каждой страте регулярное компактное слоение. С помощью [20], [21] можно сформулировать следующее следствие.

СлЕДСТВИЕ 1. Рассмотрим компактное коническое слоение $(M, \mathscr{F})$, и пусть $\mathrm{S}_{\mathscr{F}}$ есть разбиение многообразия $M$ на подмнохества, состоящие из точек слоев слоения Ғ одинаковой размерности. Если выполнено одно из следующих условий:

(i) функиия обвема слоев ограничена на каждой страте;

(ii) группа голономий слоения, идуцированного на каждую страту, конечна;

(iii) слоение на каждой страте имеет коразмерность 2;

то пространство слоев $B$ является стратифицированным псевдомногообразием и для любого искажения $\bar{p}$ имеем $\mathbb{H}_{\bar{q}}^{*}(M / \mathscr{F}) \cong \mathbb{H}_{\bar{p}}^{*}(B)$, где $\bar{q}$ есть искажсени, двойственное $\kappa \bar{p}$.

ДокАЗАТЕЛЬСтво. Условия (i), (ii) и (iii) обеспечивают то, что слоение в каждой страте является римановым. Поэтому пространство слоев $B$, снабженное стратификацией $\left\{\pi(S) \mid S \in \mathrm{S}_{\mathscr{F}}\right\}$, образует стратифицированное псевдомногообразие. На каждой страте естественная проекция является расслоением Зейферта. Осталось применить теорему 2 .

\section{5. Доказательство теоремы 1}

Разобьем доказательство на пять шагов.

1) Сведем задачу к вопросу на пространстве слоев $B$, представляя базисные когомологии пересечения слоения $(M, \mathscr{F})$, используя комплекс дифференциальных форм $\Omega_{\bar{q}}^{*}(B)$, живуших на $\pi(R)$.

2) Представим гомологии пересечения пространства $B$, используя комплекс $S C_{*}^{\bar{p}}(B)$ цепей $\bar{p}$-пересечения.

3) Поскольку эти два комплекса нельзя сравнивать с помощю интегрирования, мы вводим подкомплекс $L C_{*}^{\bar{p}}(B)$ гладких цепей $\bar{p}$-пересечения.

4) Эти комплексы сравниваются с помощью ограничения

$$
\Lambda: \operatorname{Hom}\left(L C_{*}^{\bar{p}}(B), \mathbb{R}\right) \rightarrow \operatorname{Hom}\left(S C_{*}^{\bar{p}}(B), \mathbb{R}\right)
$$

и интегрирования

$$
\Theta: \Omega_{\bar{q}}^{*}(B) \rightarrow \operatorname{Hom}\left(L C_{*}^{\bar{p}}(B), \mathbb{R}\right)
$$

5) Наконец, мы доказываем, что операторы $\Theta$ и $\Lambda$ являются квазиизоморфизмами.

5.1. Комплекс $\Omega_{\bar{q}}^{*}(B)$. То, что мы собираемся доказать, сводится к сравнению когомологий, определенных на многообразии $M$, с когомологиями, определенньми на $B$. 
Чтобы упростить доказательство, представим базисные когомологии пересечения слоения $(M, \mathscr{F})$ с помощью дифференциальных форм на $B$. Положим

$$
\Omega_{\bar{q}}^{*}(B)=\left\{\eta \in \Omega^{*}(\pi(R)) \mid \pi^{*} \eta \in \Omega_{\bar{q}}^{*}(M / \mathscr{F})\right\}
$$

Ясно, что дифференциальньй оператор $\pi^{*}: \Omega_{\bar{q}}^{*}(B) \rightarrow \Omega_{\bar{q}}^{*}(M / \mathscr{F})$ является изоморфизMOM.

Рассмотрим открытое покрытие $\{V, W\}$ пространства $B$. Существует подчиненное разбиение единицы с помощью контроллируемых функций, т.е. элементов из $\Omega \frac{0}{0}(B)$ (см. $[5])$. Значит, покрытие $\left\{\pi^{-1}(U), \pi^{-1}(V)\right\}$ является базовым, и из 3.4 .1 (b) получаем последовательность Майера-Виеториса

$$
0 \rightarrow \Omega_{\bar{q}}^{*}(B) \rightarrow \Omega_{\bar{q}}^{*}(V) \oplus \Omega_{\bar{q}}^{*}(W) \rightarrow \Omega_{\bar{q}}^{*}(V \cap W) \rightarrow 0
$$

Для того, чтобы проверить, что дифференциальная форма из $\pi(U \cap R)$ живет на $\Omega_{\bar{q}}^{*}(\pi(U))$, где $(\varphi, U)$ есть подходящая коническая карта, достаточно смотреть на $U$, а не на $\pi^{-1}(U)$. Отсюда вытекает дифференшиальньй изоморфизм

$$
\varphi^{*}: \Omega_{\bar{q}}^{*}(\pi(U)) \rightarrow \Omega_{\bar{q}}^{*}\left(\mathbb{R}^{s} \times \pi_{\mathbb{S}^{n}}\left(\mathbb{S}^{n}\right)\right)
$$

где $\pi(U)$ есть пространство слоев слоения $\left(\pi^{-1}(\pi(U)), \mathscr{F}_{\pi^{-1}(\pi(U))}\right)$, a $\mathbb{R}^{s} \times \pi_{\mathbb{S}^{n}}\left(\mathbb{S}^{n}\right)$ есть пространство слоев $\left(\mathbb{R}^{m-n-1} \times \mathbb{S}^{n}, \mathscr{H} \times c \mathscr{G}\right)$.

5.2. Комплекс $S C_{*}^{\bar{p}}(B)$. Для любого целого $i$ обозначим через $B_{i} \subset B$ объединение страт размерностей не больше $i$. Объединение сингулярных страт в $B$ обозначим через $\Sigma_{B}$. Через $\Delta$ обозначим симплекс, а через $P$ - призму (т.е. конечное произведение симплексов).

Под поднимаемой призмой c: $P \times \Delta \rightarrow B \times\left[0,1\left[^{a}\right.\right.$ будем понимать непрерьвное отображение, удовлетворяющее следующим двум условиям:

i) ограничение с : $P \times \operatorname{int}(\Delta) \rightarrow \pi(R) \times\left[0,1\left[^{a}\right.\right.$ является гладким;

ii) каждый элемент $c^{-1}\left(B_{i} \times\left[0,1\left[^{a}\right)\right.\right.$ является формой вида $P \times F_{i}$, где $F_{i}$ есть грань симплекса $\Delta$.

Поднимаемая призма с назьвается $\bar{p}$-допустимой, если она удовлетворяет условию

iii) $\operatorname{codim}_{B} \pi(S) \leqslant \operatorname{codim}_{\Delta} F_{\operatorname{dim} \pi(S)}+\bar{p}(\pi(S))$ для всех сингулярных страт $\pi(S)$.

Сингулярная цепь $\xi$ назьвается иепью $\bar{p}$-пересечения, если цепи $\xi$ и $\partial \xi$ составлены из $\bar{p}$-допустимых симплексов. Через $S C_{*}^{\bar{p}}(B)$ будем обозначать комплекс цепей $\bar{p}$-пересечений. Гомологии пересечений $\mathbb{H}_{p}^{*}(B)$ пространства $B$ можно вычислять с помощью комплекса цепей пересечения $S C_{*}^{\bar{p}}(B)$ (см. [2], [5]).

5.3. Комплекс $L C_{*}^{\bar{p}}(B)$.

5.3.1. Линейное раздутие. Рассмотрим разложсене $\Delta=\Delta_{0} * \cdots * \Delta_{k}$ симплекса $\Delta$. Можно представлять пространство $P \times \Delta$ в виде стратифицированной призмы с сингулярными стратами

$$
\left\{P \times \Delta_{0}, P \times\left(\left(\Delta_{0} * \Delta_{1}\right)-\Delta_{0}\right), \ldots, P \times\left(\left(\Delta_{0} * \cdots * \Delta_{k-1}\right)-\left(\Delta_{0} * \cdots * \Delta_{k-2}\right)\right)\right\}
$$


и регулярной стратой

$$
R_{P \times \Delta}=P \times\left(\Delta-\left(\Delta_{0} * \cdots * \Delta_{k-1}\right)\right) .
$$

Глубиной стратифищированной призмы считается $\operatorname{depth}(P \times \Delta)=k$. Для строго положительной глубины произведем десингуляризацию пространства $P \times \Delta$. Линейным раздутием пространства $P \times \Delta$ назовем гладкое отображение

$$
\mathscr{L}_{P \times \Delta}:\left(P \times \bar{c} \Delta_{0}\right) \times\left(\Delta_{1} * \cdots * \Delta_{k}\right) \rightarrow P \times \Delta
$$

определяемое по формуле

$$
\mathscr{L}_{P \times \Delta}\left(x,\left[x_{0}, t_{0}\right], y\right)=\left(x, t_{0} x_{0}+\left(1-t_{0}\right) y\right) .
$$

Здесь $\bar{c} \Delta_{0}$ обозначает замкнутьй конус $\frac{\Delta_{0} \times[0,1]}{\Delta_{0} \times\{0\}}$. Будем писать

$$
(P \times \Delta)^{\mathrm{blu}}=\left(P \times \bar{c} \Delta_{0}\right) \times\left(\Delta_{1} * \cdots * \Delta_{k}\right),
$$

причем правая часть является стратифицированной призмой, у которой

$$
\operatorname{depth}(P \times \Delta)^{\text {blu }}<\operatorname{depth}(P \times \Delta)
$$

Обратньй образ $\mathscr{L}_{P \times \Delta}^{-1}\left(R_{P \times \Delta}\right)=R_{(P \times \Delta)^{\text {blu }}}-\left(\left(P \times \Delta_{0} \times\{1\}\right) \times\left(\Delta_{1} * \cdots * \Delta_{k}\right)\right)$ плотен в $(P \times \Delta)^{\mathrm{blu}}$, а ограничение $\mathscr{L}_{P \times \Delta}: \mathscr{L}_{P \times \Delta}^{-1}\left(R_{P \times \Delta}\right) \rightarrow R_{P \times \Delta}$ является диффеоморфизмом. Те же свойства имеют место для $\operatorname{int}(P \times \Delta) \subset R_{P \times \Delta}$, где

$$
\mathscr{L}_{P \times \Delta}^{-1}(\operatorname{int}(P \times \Delta))=\operatorname{int}\left((P \times \Delta)^{\mathrm{blu}}\right) .
$$

Заметим, что если $k=1$ и $\operatorname{dim} \Delta_{1}=0$, то само отображнение $\mathscr{L}_{P \times \Delta}$ является диффеоморфизмом.

5.3.2. Гладкие гомологии пересечения. Поднимаемьй симплекс $\mathfrak{c}: \Delta \rightarrow B$ порождает естественное разложение симплнекса $\Delta$. Рассмотрим семейство индексов $\left\{i_{0}, \ldots, i_{k}\right\}$, удовлетворяюших условию $F_{i} \neq F_{i-1}$, и обозначим через $\Delta_{j}$ грань симплекса $\Delta$, для которой $F_{i_{j}}=F_{i_{j}-1}$. Этим определяется на симплексе $\Delta \mathfrak{c}$-разложение $\Delta=\Delta_{0} * \cdots * \Delta_{k}$. Тогда $\mathfrak{c}^{-1}\left(\Sigma_{B} \times\left[0,1\left[^{a}\right)=P \times\left(\Delta_{0} * \cdots * \Delta_{k-1}\right)\right.\right.$.

Призма назьвается гладкой, если вьполняется условие

iv) существует гладкое отображение $\mathfrak{c}: P \times \Delta \rightarrow M \times\left[0,1\left[^{a}\right.\right.$ такое, что $\pi \circ \mathfrak{c}=\mathfrak{c}$.

Поскольку с ${ }^{-1}\left(\pi(R) \times\left[0,1\left[^{a}\right)=R_{P \times \Delta}\right.\right.$, то ограничение $\mathfrak{c}: R_{P \times \Delta} \rightarrow R \times\left[0,1\left[^{a}\right.\right.$ является гладким, и поэтому вьполняется более сильное, чем і) условие, а именно

$\left.\mathrm{i}^{\prime}\right)$ ограничение $\mathfrak{c}: R_{P \times \Delta} \rightarrow \pi(R) \times\left[0,1{ }^{a}\right.$ является гладким.

Сингулярная цепь $\xi$ называется гладкой иепью $\bar{p}$-пересечения, если цепи $\xi$ и $\partial \xi$ состоят из гладких $\bar{p}$-допустимых симплексов. Через $L C_{*}^{\bar{p}}(B)$ обозначим комплекс гладких цепей $\bar{p}$-пересечения. 
5.4. Операторы $\Theta$ и $\Lambda$. Естественное вложение $L C_{*}^{\bar{p}}(B) \hookrightarrow S C_{*}^{\bar{p}}(B)$ индуцирует диффренциальньй оператор

$$
\Lambda: \operatorname{Hom}\left(S C_{*}^{\bar{p}}(B), \mathbb{R}\right) \rightarrow \operatorname{Hom}\left(S C_{*}^{\bar{p}}(B), \mathbb{R}\right) .
$$

Трудность интегрирования дифференциальной формы $\omega \in \Omega_{q}^{*}(B)$ по цепи пересечения $\xi$ заключается в том факте, что $\omega$ определена только на регулярной страте пространства $B$, в то время как $\xi$ определена на всем $B$. По этой причине нам необходимы некоторые предварительные результаты. Линейное раздутие совместимо с барицентрическим подразделением. Пусть $\nabla$ - элемент барицентрического подразделения симплекса $\Delta$. В $[22$, c. 220] доказано следуюшее

ПРЕДЛОЖЕНИЕ 7. Для данного әлемента $\nabla$ баричентрического подразделения симплекса $\Delta$ и естественного вложения $I:(P \times \nabla)^{\mathrm{blu}} \hookrightarrow(P \times \Delta)^{\mathrm{blu}}$ существует гладкое отображение $I: P \times \nabla \rightarrow P \times \Delta$, удовлетворяющее условию $I \circ \mathscr{L}_{P \times \nabla}=$ $\mathscr{L}_{P \times \Delta} \circ I$.

5.4.1. Дифференциальные формы на $P \times \Delta$. Через $\Pi^{*}(P \times \Delta)$ обозначим комплекс поднимаемых форм. Если $\operatorname{depth}(P \times \Delta)=0$, то полагаем $\Pi^{*}(P \times \Delta)=\Omega^{*}(P \times \Delta)$. В общем случае скажем, что дифференциальная форма $\omega \in \Omega^{*}\left(R_{P \times \Delta}\right)$ поднимаема, если существует поднимаемая форма $\widehat{\omega} \in \Pi^{*}\left(P \times \Delta^{\text {blu }}\right)$, для которой $\mathscr{L}_{P \times \Delta}^{*} \omega=\widehat{\omega}$ на $\mathscr{L}_{P \times \Delta}^{-1}\left(R_{P \times \Delta}\right)$. Заметим, что, если $k=1$ и $\operatorname{dim} \Delta_{1}=0$, то форма $\omega$ в действительности определена на $P \times \Delta$. В силу плотности поднятие $\widehat{\omega}$ единственно. Тогда $\widehat{d \omega}=d \widehat{\omega}$. Значит, комплекс $\Pi^{*}(P \times \Delta)$ дифференциальньй. Как обычно, будем писать

$$
\int_{P \times \Delta} \omega=\int_{\operatorname{int}(P \times \Delta)} \omega
$$

хотя это не всегда корректно определено. Но в нашем случае имеет место

Лемма А. Если $\omega-m$-поднимаемая форма, то интеграл $\int_{P \times \Delta} \omega$ конечен.

ДокАЗАТЕЛЬСтво. Если глубина равна 0 , то утверждение очевидно. В общем случае

$$
\int_{P \times \Delta} \omega=\int_{\mathscr{L}_{P \times \Delta}^{-1}(\operatorname{int}(P \times \Delta))} \mathscr{L}_{P \times \Delta}^{*} \omega=\int_{\operatorname{int}(P \times \Delta)} \widehat{\omega}=\int_{P \times \Delta} \widehat{\omega}
$$

в силу (4) и $\operatorname{int}(P \times \Delta) \subset R_{P \times \Delta}$. По индукционному предположению это число конечно.

Лемма В. Пусть $\mathfrak{c}: P \times \Delta \rightarrow B \times\left[0,1\left[^{a}-\right.\right.$ гладкая призма. Если $\eta \in \Omega^{*}(\pi(R) \times$ $\left[0,1\left[^{a}\right) u \pi^{*} \eta \in \Pi^{*}\left(M \times\left[0,1\left[^{a}\right)\right.\right.\right.$, то $\mathfrak{c}^{*} \eta$ поднимаемо.

ДокАЗАТЕЛЬСтво. Поскольку $\mathfrak{c}^{*} \eta \in \Omega^{*}\left(R_{P \times \Delta}\right)($ см. і') $)$, достаточно построить поднятие $\widehat{\mathfrak{c}^{*} \eta} \in \boldsymbol{\Pi}^{*}(P \times \Delta)$. Доказательство проведем в несколько шагов.

I) Локализация пространств $M$ и $B$. Заметим, что для любого элемента $\nabla$ барицентрического подразделения симплекса $\Delta$ ограничение $\mathfrak{c}^{\prime}: P \times \nabla \rightarrow B \times\left[0,1\left[{ }^{a}\right.\right.$ является гладкой призмой. Это утверждение локально. Поэтому мы можем идентифицировать $(M, \mathscr{F}) \equiv\left(\mathbb{R}^{r} \times \mathbb{R}^{s} \times c \mathbb{S}^{n}, \mathscr{K} \times \mathscr{I} \times c \mathscr{G}\right), B \equiv \mathbb{R}^{s} \times c \pi_{\mathbb{S}^{n}}\left(\mathbb{S}^{n}\right)$ и предположить, что $\operatorname{Im} \mathfrak{c}$ 
meets $\mathbb{R}^{\mathrm{blu}} \times\{\vartheta\} \times\left[0,1\left[^{a}\right.\right.$. Тогда $\mathrm{c}^{-1}\left(\mathbb{R}^{s} \times\{\vartheta\} \times\left[0,1\left[^{a}\right)=P \times \Delta_{0}\right.\right.$. Отметим, что окрестность $\Delta_{0}$ в симплексе $\Delta$ является произведением $\Delta_{0} \times c \nabla$, где $\nabla$ - некоторьй симплекс. Из леммы 1 получаем коммутативную диаграмму

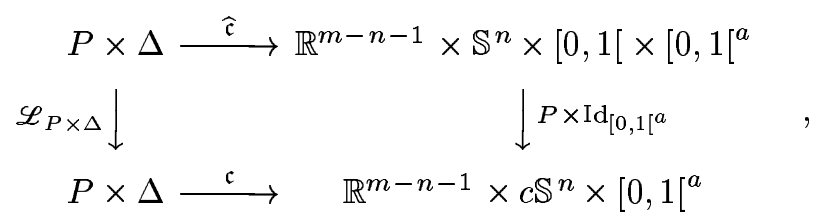

где $\widehat{\mathfrak{c}}$ гладко.

II) Раздутие с. Рассмотрим теперь непрерьвное отображение

$$
\widehat{\mathfrak{c}}=\widehat{\pi} \circ \widehat{\mathfrak{c}}: P \times \Delta \rightarrow \mathbb{R}^{s} \times \pi_{\mathbb{S}^{n}}\left(\mathbb{S}^{n}\right) \times\left[0,1\left[^{a+1},\right.\right.
$$

где $\widehat{\pi}: \mathbb{R}^{m-n-1} \times \mathbb{S}^{n} \times\left[0,1\left[^{a+1} \rightarrow \mathbb{R}^{s} \times \pi_{\mathbb{S}^{n}}\left(\mathbb{S}^{n}\right) \times\left[0,1\left[^{a+1}\right.\right.\right.\right.$ есть проекция, определяемая по формуле

$$
\widehat{\pi}\left(x_{1}, \ldots, x_{r}, y_{1}, \ldots, y_{s}, \theta, t_{0}, t_{1}, \ldots, t_{a}\right)=\left(y_{1}, \ldots, y_{s}, \pi_{\mathbb{S}^{n}}(\theta), t_{0}, t_{1}, \ldots, t_{a}\right) .
$$

Покажем, что $\widehat{\mathfrak{c}}$ является гладкой призмой.

i) Поскольку $\widehat{\pi}$ и $\widehat{\mathfrak{c}}$ являются гладкими, достаточно проверить, что

$$
\widehat{\mathfrak{c}}^{-1}\left(\mathbb{R}^{s} \times \pi_{\mathbb{S}^{n}}\left(\Sigma_{\mathscr{G}}\right) \times\left[0,1\left[^{a+1}\right) \subset\left(\left(P \times \bar{c} \Delta_{0}\right) \times\left(\left(\Delta_{1} * \cdots * \Delta_{k}\right)-\operatorname{int}\left(\Delta_{1} * \cdots * \Delta_{k}\right)\right)\right) .\right.\right.
$$

Это следует из того, что

$$
\begin{aligned}
\widehat{\mathfrak{c}}^{-1} \widehat{\pi}^{-1}\left(\mathbb{R}^{s} \times \pi_{\mathbb{S} n}\left(\Sigma_{\mathscr{G}}\right) \times\left[0,1\left[^{a+1}\right)\right.\right. \\
=\mathscr{L}_{P \times \Delta}^{-1}\left(P \times\left(\Delta_{0} * \cdots * \Delta_{k-1}\right)\right. \\
\quad \subset\left(\left(P \times \bar{c} \Delta_{0}\right) \times\left(\left(\Delta_{1} * \cdots * \Delta_{k}\right)-\operatorname{int}\left(\Delta_{1} * \cdots * \Delta_{k}\right)\right)\right)
\end{aligned}
$$

ii) Проводя аналогичные рассуждения, мы получаем

$$
\widehat{\mathfrak{c}}^{-1}\left(\mathbb{R}^{s} \times\left(\pi_{\mathbb{S}^{n}}\left(\mathbb{S}^{n}\right)\right)_{j} \times\left[0,1\left[^{a+1}\right)=\mathscr{L}_{P \times \Delta}^{-1}\left(P \times\left(\Delta_{0} * \cdots * \Delta_{h}\right)\right)=P \times\left(\Delta_{0} * \cdots * \Delta_{h}\right)\right.\right.
$$

для подходящего $h \in\{1, \ldots, k\}$.

iii) На основании построения.

III) Поднятие $\eta$. Дифференциальная форма $\pi^{*} \eta \in \boldsymbol{\Pi}^{*}\left(\mathbb{R}^{m-n-1} \times a^{n} \times\left[0,1\left[^{a}\right)\right.\right.$ поднимается до дифференциальной формы $\widehat{\pi^{*} \eta} \in \Pi^{*}\left(\mathbb{R}^{m-n-1} \times \mathbb{S}^{n} \times\left[0,1\left[^{a+1}\right)\right.\right.$. Поскольку форма $\pi^{*} \eta$ является базисной, а ограничение отображения $P \times \operatorname{Id}_{[0,1[}{ }^{a}$ на $\mathbb{R}^{m-n-1} \times R_{0} \times$ ] $0,1\left[\times\left[0,1\left[^{a}\right.\right.\right.$, где $R_{0}$ есть регулярная страта в $\mathbb{S}^{m-n-1}$, является тождественным отображением, то форма $\widehat{\pi^{*} \eta}$ тоже является базисной. Существует форма $\widehat{\eta} \in \Omega^{*}\left(\mathbb{R}^{s} \times\right.$ $\pi_{\mathbb{S}^{n}}\left(R_{0}\right) \times\left[0,1\left[^{a+1}\right)\right.$ такая, что $\widehat{\pi}^{*} \widehat{\eta}=\widehat{\pi^{*} \eta}$. Тогда дифференшиальная форма $\widehat{\eta}$ удовлетворяет условиям леммы.

IV) Заключительный шаг. Проведем индукцию по глубине depth $S_{\mathscr{F}}$. Результат очевиден, когда глубина равна 0 , т.е., когда $B=\pi(R)$. В общем случае заметим, что из неравенства depth $\mathrm{S}_{\mathscr{H}} \times \mathscr{G}<\operatorname{depth} \mathrm{S}_{\mathscr{H}} \times c_{\mathscr{G}}$ следует по предположению индукции, что 
форма $\widehat{\mathfrak{c}}^{*} \widehat{\eta}$ поднимаема. Осталось установить, что $\widehat{\mathfrak{c}^{*} \eta}=\widehat{\mathbf{c}}^{*} \widehat{\eta}$, т.е., что $\mathscr{L}_{P \times \Delta}^{*} \mathfrak{c}^{*} \eta=\widehat{\mathbf{c}}^{*} \widehat{\eta}$ на $\mathscr{L}_{P \times \Delta}^{-1}\left(R_{P \times \Delta}\right)$. Поскольку

$$
\mathfrak{c} \circ \mathscr{L}_{P \times \Delta}=\left(P \times \operatorname{Id}_{\left[0,1\left[{ }^{a}\right.\right.}\right) \circ \widehat{\mathfrak{c}},
$$

TO

$$
\mathscr{L}_{P \times \Delta}^{*} \mathfrak{c}^{*} \eta=\mathscr{L}_{P \times \Delta}^{*} \mathfrak{c}^{*} \pi^{*} \eta=\widehat{\mathfrak{c}}^{*}\left(P \times \operatorname{Id}_{\left[0,1\left[{ }^{a}\right.\right.}\right)^{*} \pi^{*} \eta
$$

на $\mathscr{L}_{P \times \Delta}^{-1}\left(R_{P \times \Delta}\right)$. Поскольку форма $\pi^{*} \eta$ поднимаема, то

$$
\left(P \times \operatorname{Id}_{\left[0,1\left[{ }^{a}\right.\right.}\right)^{*} \eta=\widehat{\pi^{*} \eta}=\widehat{\pi}^{*} \widehat{\eta}
$$

на $\left.\mathbb{R}^{m-n-1} \times\left(\mathbb{S}^{n}-\Sigma_{\mathbb{S}^{n}}\right) \times\right] 0,1\left[\times\left[0,1\left[{ }^{a}\right.\right.\right.$.

Таким образом,

$$
\mathscr{L}_{P \times \Delta}^{*} \mathfrak{c}^{*} \eta=\widehat{\mathfrak{c}}^{*} \widehat{\pi}^{*} \hat{\eta}=\widehat{\mathbf{c}}^{*} \widehat{\eta}
$$

на $\widehat{\mathfrak{c}}^{-1}\left(\mathbb{R}^{m-n-1} \times\left(\mathbb{S}^{n}-\Sigma_{\mathbb{S}^{n}}\right) \times\right] 0,1\left[\times\left[0,1\left[{ }^{a}\right)=\mathscr{L}_{P \times \Delta}^{-1}\left(R_{P \times \Delta}\right)\right.\right.$.

Для заданной формы $\eta \in \Omega_{\bar{p}}^{*}(B)$ и гладкого $\bar{p}$-допустимого симплекса $c: \Delta \rightarrow B$ можно определить интеграл

$$
\Theta(\omega)(c)=\int_{\Delta} c^{*} \eta
$$

(см. лемму А и лемму В). Осталось доказать, что оператор

$$
\Theta: \Omega_{\bar{q}}^{*}(B) \rightarrow \operatorname{Hom}\left(L C_{*}^{\bar{p}}(B), \mathbb{R}\right)
$$

является дифференциальным. Нам нужны некоторые предварительные результаты.

5.4.2. Гранища. Имеется два типа граней (коразмерности 1$)$ на симплексе $(P \times \Delta)^{\mathrm{blu}}$ :

Т1) грани $(Q \times \nabla)^{\text {blu }}$, где $Q$ есть грань $P$, a $\nabla=\Delta$, или $Q=P$ и $\nabla$ есть грань $\Delta$; ограничение $\mathscr{L}_{P \times \Delta}$ является линейньм раздутием $\mathscr{L}_{Q \times \nabla}$;

Т2) грань $F_{P \times \Delta}=\left(P \times \Delta_{0} \times\{1\}\right) \times\left(\Delta_{1} * \cdots * \Delta_{k}\right)$; ограничение $\mathscr{L}_{P \times \Delta}$ в точности есть каноническая проекция на $P \times \Delta_{0}$.

Грани типа T1) расположены над границей $P \times \Delta$. Грань же $F_{P \times \Delta}$ является дополнительной гранью, возникающей в результате раздутия. Имеет место формула

$$
\partial\left((P \times \Delta)^{\mathrm{blu}}\right)=(\partial(P \times \Delta))^{\mathrm{blu}}+F_{P \times \Delta} .
$$

5.4.3. Дополнительные диффернециальные формы на $P \times \Delta$. Через $\Gamma^{*}(P \times \Delta)$ обозначим комплекс регулярных форм. Если $\operatorname{depth}(P \times \Delta)=0$, то полагаем

$$
\Gamma^{*}(P \times \Delta)=\Omega^{*}(P \times \Delta) .
$$

В общем случае скажем, что поднимаемая форма $\omega \in \boldsymbol{\Pi}^{*}(P \times \Delta)$ регулярна, если $\widehat{\omega} \in \Gamma^{*}(P \times \Delta)$ и ее ограничение на int $F_{P \times \Delta}$ равно 0. Заметим, что $\boldsymbol{\Gamma}^{*}(P \times \Delta)$ является дифференциальным комплексом. Из предложения 7 следует, что ограничение регулярной формы на элемент барицентрического подразделения остается регулярным. Для этого вида форм имеет место следующая формула Стокса. 
Лемма С. Пусть $\omega-$ регулярная форма. Тогда

$$
\int_{P \times \Delta} d \omega=\int_{\partial(P \times \Delta)} \omega
$$

ДоКАЗАТЕЛЬСТво проведем индукцией по глубине. Если глубина равна 0 , то утверждение очевидно. В общем случае заметим, что форма $\omega$ определена на внутренности любой грани $F$ пространства $P \times \Delta$ кроме случая, когда $\operatorname{dim} \Delta_{k}=0$ и $F=\Delta_{0} * \cdots * \Delta_{k-1}$. Но в этом сдучае форма $\omega$ продолжается до формы на int $F$. Чтобы усмотреть это, применим предложение 7 , чтобы свести задачу к симплексу $\Delta=\Delta_{0} * \Delta_{1}$ и $\operatorname{dim} \Delta_{1}=0$. Отображение $\mathscr{L}_{P \times \Delta}$ является диффеоморфизмом, а форма $\widehat{\omega}$ определена всюду; значит, и форма $\omega$ всюду определена. Следовательно, оба члена рассматриваемого равенства имеют смысл. По предположению индукции имеем

$$
\int_{P \times \Delta} \widehat{d \omega}=\int_{\partial(P \times \Delta)} \widehat{\omega}
$$

и поэтому можно применить $(7)$ и (5) и заметить, что форма $\widehat{\omega}$ тривиальна на $F_{P \times \Delta}$.

Лемма D. Пусть $\mathfrak{c}: P \times \Delta \rightarrow B \times\left[0,1\left[{ }^{a}\right.\right.$ есть гладкая $\bar{p}$-допустимая призма. Если $\eta \in \Omega_{q}^{*}(B)$, то форма $\mathfrak{c}^{*} \eta$ регулярна.

ДокАЗАТЕЛЬСтво. Поскольку форма $\mathfrak{c}^{*} \eta$ поднимаема, нужно только установить, что форма $\widehat{\mathfrak{c}^{*} \eta}$ тривиальна на int $F_{P \times \Delta}$. Действительно, достаточно доказать, что

$$
\widehat{\mathfrak{c}}^{*} \widehat{\eta}\left(u_{1}, \ldots, u_{a}, v_{1}, \ldots, v_{b}, 0, w_{1}, \ldots w_{c}\right)=0
$$

где $\left\{u_{1}, \ldots, u_{a=\operatorname{dim} P}\right\}$ - касательные векторы к $\operatorname{int} P,\left\{v_{1}, \ldots, v_{b=\operatorname{dim}} \Delta_{0}\right\}$-касательные векторы к int $\Delta_{0}$, a $\left\{w_{1}, \ldots, w_{c=\operatorname{dim}} \Delta_{1} * \cdots * \Delta_{k}\right\}$ - касательные векторы к $\operatorname{int}\left(\Delta_{1} * \cdots * \Delta_{k}\right)$.

Поскольку проблема является локальной, как и прежде, отождествим $(M, \mathscr{F}) \mathrm{c}\left(\mathbb{R}^{r} \times\right.$ $\left.\mathbb{R}^{s} \times c \mathbb{S}^{n}, \mathscr{K} \times \mathscr{I} \times c \mathscr{G}\right), B \mathrm{c} \mathbb{R}^{s} \times c \pi_{\mathbb{S}^{n}}\left(\mathbb{S}^{n}\right), S \mathrm{c} \mathbb{R}^{m-n-1} \times\{\vartheta\}$ и предположим, что $\operatorname{Im} \mathfrak{c}$ пересекает $\mathbb{R}^{\mathrm{blu}} \times\{\vartheta\} \times\left[0,1\left[^{a}\right.\right.$. Тогда $\mathfrak{c}^{-1}\left(\mathbb{R}^{s} \times\{\vartheta\} \times\left[0,1\left[^{a}\right)=P \times \Delta_{0}\right.\right.$. Тогда диаграмма (6) примет вид

$$
\begin{array}{ccc}
F_{P \times \Delta} \stackrel{\widehat{\mathfrak{c}}}{\longrightarrow} \mathbb{R}^{m-n-1} \times \mathbb{S}^{n} \times\{0\} \times\left[0,1\left[^{a}\right.\right. \\
\mathscr{L}_{P \times \Delta} \downarrow & & \\
& & P \times \operatorname{Id}_{\left[0,1\left[^{a}\right.\right.} \\
P \times \Delta_{0} \stackrel{\mathfrak{c}}{\longrightarrow} & \mathbb{R}^{m-n-1} \times\{\vartheta\} \times\left[0,1\left[^{a}\right.\right.
\end{array}
$$

Здесь $\mathscr{L}_{P \times \Delta}\left(x_{1}, \ldots, x_{a}, y_{1}, \ldots, y_{b}, 0, z_{1}, \ldots, z_{c}\right)=\left(x_{1}, \ldots, x_{a}, y_{1}, \ldots, y_{b}\right)$. Призма $\widehat{\mathfrak{c}}$ удовлетворяет условию і'), и поэтому отображение $\widehat{\mathfrak{c}}$ отображает int $F_{P \times \Delta}$ в $\mathbb{R}^{m-n-1} \times$ $\left(\mathbb{S}^{n}-\Sigma \mathscr{G}\right) \times\{0\} \times\left[0,1\left[^{a}\right.\right.$. Поскольку $\widehat{\mathfrak{c}}=\widehat{\pi} \circ \widehat{\mathfrak{c}}$ и $\widehat{\pi}^{*} \widehat{\eta}=\widehat{\pi^{*} \eta}$, условие (8) эквивалентно

$$
\widehat{\pi^{*} \eta}\left(\widehat{\mathfrak{c}}_{*} u_{1}, \ldots, \widehat{\mathbf{c}}_{*} u_{a}, \widehat{\mathfrak{c}}_{*} v_{1}, \ldots, \widehat{\mathfrak{c}}_{*} v_{b}, 0, \widehat{\mathbf{c}}_{*} w_{1}, \ldots \widehat{\mathfrak{c}}_{*} w_{c}\right)=0
$$

Поскольку

$$
\operatorname{codim}_{\Delta} F_{\operatorname{dim} \pi(S)}=\operatorname{codim}_{\Delta} F_{s}=\operatorname{codim}_{\Delta} \Delta_{0}=c+1,
$$


из условия iv) следует, что

$$
c \geqslant \operatorname{codim}_{B} \pi(S)-\bar{p}(\pi(S))-1=\bar{q}(\pi(S))+1>\bar{q}(\pi(S)) .
$$

Наконец, поскольку $\left(\mathscr{L}_{P \times \Delta}\right)_{*} w_{j}=0$, вектор $\widehat{\mathfrak{c}}_{*} w_{j}$ принадлежит пространству $\mathbb{R}^{m-n-1} \times\left(\mathbb{S}^{n}-\Sigma_{\mathscr{G}}\right) \times\{0\} \times\left[0,1\left[^{a}\right.\right.$, касающемуся слоев $\left(P \times \operatorname{Identity~}_{[0,1}{ }^{a}\right)$, и поэтому получаем результат.

Леммы С и $\mathrm{D}$ показьвают, что оператор $\Theta: \Omega_{\bar{q}}^{*}(B) \rightarrow \operatorname{Hom}\left(L C_{*}^{\bar{p}}(B), \mathbb{R}\right)$ является дифференциальным оператором.

5.5. Квазиизоморфизмы $\Theta$ и $\Lambda$. Рассмотрим утверждение $Q(B)$ о пространстве слоев сингулярного расслоения:

$$
\begin{gathered}
Q(B)=“ \Omega_{\bar{q}}^{*}(B) \stackrel{\Theta}{\rightarrow} \operatorname{Hom}\left(L C_{*}^{\bar{p}}(B), \mathbb{R}\right) \stackrel{\Lambda}{\leftarrow} \operatorname{Hom}\left(S C_{*}^{\bar{p}}(B), \mathbb{R}\right) \\
\text { являются квазиизоморффизмами", }
\end{gathered}
$$

и докажем его, используя трюк Брэдона [23, с. 289].

ТрЮк БРЭдонА. Пусть $X$ - паракомпактное топологическое пространство, $\left\{U_{\alpha}\right\}$ - открытое покрытие, замкнутое относительно конечных пересечений. Предположим, что $Q(U)$ есть утверждение об открытых подмножествах пространства $X$, удовлетворяюших следующим трем свойствам:

(ВТ1) утверждение $Q\left(U_{\alpha}\right)$ справедливо для любого $\alpha$;

(ВТ2) из справедливости утверждений $Q(U), Q(V)$ и $Q(U \cap V)$ следует справедливость утверждения $Q(U \cup V)$, где $U$ и $V$ - открытые подмнохества пространства $X$;

(ВТ3) из справедливости утверждений $Q\left(U_{i}\right)$ следует справедливость утверждения $Q\left(\bigcup_{i} U_{i}\right)$, где $\left\{U_{i}\right\}$ есть дизбюнктное семейство открытых подмножеств в $X$.

Тогда утверждение $Q(X)$ справедливо.

Проведем доказательство индукцией по глубине depth $\mathrm{S}_{\mathscr{F}}$. Если глубина равна 0 , то утверждение $Q(B)$ есть обычная теорема де Рама. Предположим, что утверждение $Q(B)$ доказано, когда depth $\mathrm{S}_{\mathscr{F}}<\ell$. Далле рассмотрим несколько шагов.

(i) Пусть слоение $(M, \mathscr{F})$ равно $\left(\mathbb{R}^{u} \times \mathbb{R}^{v} \times c S^{w}, \mathscr{K} \times \mathscr{I} \times c \mathscr{G}\right)$, где $\mathscr{K}$ есть слоение с одним слоем, $\mathscr{I}$ есть слоение, состоящее из точек, и $\left(S^{v}, \mathscr{G}\right)$ есть сингулярное слоение, для которогъо $\operatorname{depth} \mathscr{S}_{\mathscr{G}}<\ell$.

Используя каноническую проекшию $\operatorname{Pr}: \mathbb{R}^{v} \times c \pi_{S w}\left(S^{w}\right) \rightarrow c \pi_{S}\left(S^{w}\right)$, ограниченную на регулярную часть, получаем, что $\operatorname{Pr}^{*}: \Omega_{\bar{q}}^{*}\left(c \pi_{S^{w}}\left(S^{w}\right)\right) \rightarrow \Omega_{\bar{q}}^{*}\left(\mathbb{R}^{v} \times c \pi_{S^{w}}\left(S^{w}\right)\right)$ есть квазиизоморфизм (см. предложение 2 и (6)). Следуя рассуждениям работы [22], доказьваем, что операторы

$$
\operatorname{Pr}^{*}: \operatorname{Hom}\left(S C_{*}^{\bar{p}}\left(c \pi_{S^{w}}\left(S^{w}\right)\right), \mathbb{R}\right) \rightarrow \operatorname{Hom}\left(S C_{*}^{\bar{p}}\left(\mathbb{R}^{v} \times c \pi_{S^{w}}\left(S^{w}\right)\right), \mathbb{R}\right)
$$

и

$$
\operatorname{Pr}^{*}: \operatorname{Hom}\left(L C_{*}^{\bar{p}}\left(c \pi_{S^{w}}\left(S^{w}\right)\right), \mathbb{R}\right) \rightarrow \operatorname{Hom}\left(L C_{*}^{\bar{p}}\left(\mathbb{R}^{v} \times c \pi_{S^{w}}\left(S^{w}\right)\right), \mathbb{R}\right)
$$


являются квазиизоморфизмами. Поскольку отображение $\operatorname{Pr}^{*}$ коммутирует с $\Theta$ и $\Lambda$, то

$$
Q\left(c \pi_{S w}\left(S^{w}\right)\right) \Longrightarrow Q\left(\mathbb{R}^{v} \times c \pi_{S w}\left(S^{w}\right)\right)
$$

Остается доказать утверждение $Q\left(c \pi_{S^{w}}\left(S^{w}\right)\right)$.

Из предложения 3 и (6) следует, что каноническая проекция

$$
\left.\operatorname{Pr}:\left(S^{w}-\Sigma_{\mathscr{G}}\right) \times\right] 0,1\left[\rightarrow\left(\mathbb{S}^{n}-\Sigma_{\mathscr{G}}\right)\right.
$$

индуцирует квазиизоморфизм

$$
\mathbb{H}_{\bar{q}}^{i}\left(c \pi_{S w}\left(S^{w}\right)\right)= \begin{cases}\mathbb{H}_{\bar{p}}^{i}\left(\pi_{S^{w}}\left(S^{w}\right)\right) & \text { при } i \leqslant \bar{q}(\{\vartheta\}), \\ 0 & \text { при } i>\bar{q}(\{\vartheta\}) .\end{cases}
$$

Следуя рассуждениям работы [22], доказываем, что отображение $\mathrm{Pr}^{*}$ индуцирует квазиизоморфизмы

$$
H^{i}\left(L C_{*}^{\bar{p}}\left(c \pi_{S^{w}}\left(S^{w}\right)\right)= \begin{cases}H^{i}\left(L C_{*}^{\bar{p}}\left(\pi_{S^{w}}\left(S^{w}\right)\right)\right. & \text { при } i \leqslant k-1-\bar{p}(\{\vartheta\}), \\ 0 & \text { при } i>k-1-\bar{p}(\{\vartheta\}),\end{cases}\right.
$$

и

$$
H^{i}\left(S C_{*}^{\bar{p}}\left(c \pi_{S^{w}}\left(S^{w}\right)\right)= \begin{cases}H^{i}\left(S C_{*}^{\bar{p}}\left(\pi_{S^{w}}\left(S^{w}\right)\right)\right. & \text { при } i \leqslant k-1-\bar{p}(\{\vartheta\}), \\ 0 & \text { при } i>k-1-\bar{p}(\{\vartheta\}),\end{cases}\right.
$$

где $k=\operatorname{dim} \pi_{S^{w}}\left(S^{w}\right)$. Далее, поскольку $\Theta$ и $\Lambda$ коммутируют с $\operatorname{Pr}^{*}$, достаточно применить утверждение $Q\left(\pi_{S} w\left(S^{w}\right)\right)$ и принять во внимание, что искажения $\bar{p}$ и $\bar{q}$ на пространстве $B$ двойственны.

(ii) Пусть слоение $(M, \mathscr{F})$ является открытым подмножеством в $\left(\mathbb{R}^{u} \times \mathbb{R}^{v} \times\right.$ $\left.c S^{w}, \mathscr{K} \times \mathscr{I} \times c \mathscr{G}\right)$, где $\mathscr{K}$ есть слоение с одним слоем, $\mathscr{I}$ есть слоение, состоящее из точек, а $\left(S^{v}, \mathscr{G}\right)$ есть сингулярное расслоение глубины $\operatorname{depth} \mathrm{S}_{\mathscr{G}}<\ell$.

Поскольку пространство $B$ паракомпактно, то применяем трюк Брэдона к покрытию

$$
\begin{gathered}
\left\{V \times c_{\varepsilon} \pi_{S w}\left(S^{w}\right) \mid V=\right] a_{1}, b_{1}[\times \cdots \times] a_{v}, b_{v}\left[\subset \mathbb{R}^{v}, \varepsilon \in[0,1[\}\right. \\
\cup\left\{\pi(U) \mid U \subset \mathbb{R}^{u+v} \times S^{w} \times\right] 0,1[\text { открыто }\},
\end{gathered}
$$

где $c_{\varepsilon} \pi_{S w}\left(S^{w}\right)=\pi_{S w}\left(S^{w}\right) \times\left[0, \varepsilon\left[/ \pi_{S w}\left(S^{w}\right) \times\{0\}\right.\right.$. Это семейство замкнуто по отношению к конечным пересечениям. Проверка условий (ВТ1)-(ВТ3) очевидна.

(iii) Пусть глубина слоения $(M, \mathscr{F})$ равна $\ell$.

Поскольку пространство $B$ паракомпактно, применим трюк Брэдона к следующему покрытию:

$$
\begin{gathered}
\{V \subset B \mid V \text { открытое подмножество в } \pi(U), \\
\text { где }(\varphi, U) \text { есть хорошая коническая карта }\} .
\end{gathered}
$$

Это семейство замкнуто по отношению к конечньм пересечениям и вьполняются условия (ВТ1)-(ВТ3). 


\section{СПИСОК ЦИТИРОВАННОЙ ЛИТЕРАТУРЫ}

[1] Goresky M., MacPherson R. Intersection homology theory // Topology. 1980. V. 19. P. 135-162.

[2] King H. Topology invariance of intersection homology without sheaves // Topology Appl. 1985. V. 20. P. 149-160.

[3] MacPherson R. Intersection homology and perverse sheaves // Colloquium Lectures. Annual Meeting of AMS. San Francisco, June 1991.

[4] Brylinsky J. L. Equivariant intersection cohomology // Contemp. Math. 1992. V. 132. P. 5-32.

[5] Saralegi M. Homological properties of stratified spaces // Illinois J. Math. 1994. V. 38. P. 47-70.

[6] Verona A. Le thèoréme de Rham pour les préstratifications abstraites // C. R. Acad. Sci. Paris. 1971. V. 273. P. 886-889.

[7] Saralegi M., Wolak R. Basic intersection cohomology for singular riemanian foliations. Preprint.

[8] Molino P. Riemannian Foliations // Progress in Math. V. 73. Basel: Birkhäuser, 1988.

[9] Wolak R. Pierrot's theorem for singular Riemannian foliations // Publ. Matem. 1994. V. 38. P. 433-439.

[10] Bauer M. Feuilletage singulier défini par une distribution presque régulière // Collect. Math. 1986. V. 37. № 3. P. 189-209.

[11] Stefan P. Accesible sets, orbits, and foliations with singularities // Proc. London Math. Soc. 1974. V. 29. P. 699-713.

[12] Sussmann H. J. Orbit of families of vector fields and integrability of distributions // Trans. Amer. Math. Soc. 1973. V. 180. P. 171-188.

[13] Vaisman I. Lectures on the Geometry of Poisson Manifolds // Progress in Math. V. 118. Basel: Birkhäser, 1994.

[14] Вифлянцев В. П. Теорема Фробениуса для распределений с особенностями // УМН. 1977. Т. 32 . № 5. С. $177-178$.

[15] Вифлянцев В.П. Теорема Фробениуса для дифференциальных систем с особенностями // Вестн. МГУ. Сер. 1. Матем., мех. 1980. Т. 106. № 3. С. 11-14.

[16] Davis M. Smooth G-manifolds as collections of fibre bundles // Pacific J. Math. 1978. V. 77. P. $315-363$.

[17] Nguyen Tien Zung Symplectic topology of integrable Hamiltonian systems, II: Topological classification. Preprint: Universitè Montpellier II, 2001.

[18] Boualem H., Molino P. Modeles locaux satures de feuilletages Riemanniens singuliers // C. R. Acad. Sci. Paris. 1993. V. 316. P. 913-916.

[19] Bredon G. E. Introduction to Compact Transformation Groups // Pure Appl. Math. V. 46. New York: Acad. Press, 1972.

[20] Epstein D. B. A. Foliations with all leaves compact // Ann. Inst. Fourier (Grenoble). 1976. V. 26. P. $265-282$.

[21] Edwards R. D., Millet K. C., Sullivan D. Foliations with all leaves compact // Topology. 1977. V. 16. P. 13-32.

[22] Brasselet J. P., Hector G., Saralegi M. Theoreme de Rham pour les varietes stratifiees // Ann. Global Anal. Geom. 1991. V. 9. P. 211-243.

[23] Bredon G.E. Topology and Geometry // Graduate Text in Math. V. 139. Berlin: Springer, 1993.

(М. Саралеги-Арангурен) Университет д'Артуа, Франция

Поступило

(Р. Волак) Ягеллонский университет, г. Краков, Польша

17.09.2002 